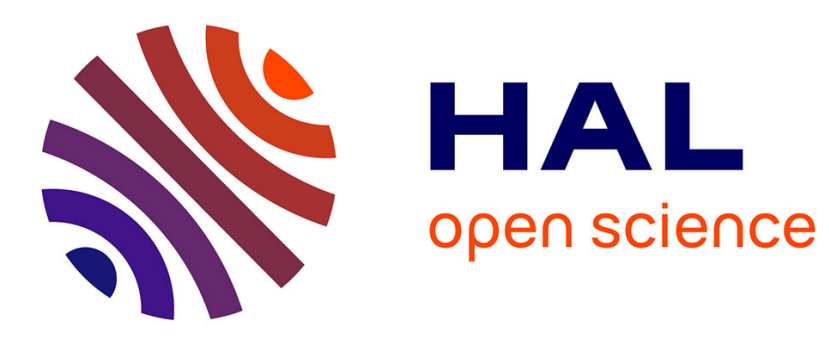

\title{
Interface debonding models: a viscous regularization with a limited rate dependency
}

Jean Louis L Chaboche, Frédéric Feyel, Yann Monerie

\section{To cite this version:}

Jean Louis L Chaboche, Frédéric Feyel, Yann Monerie. Interface debonding models: a viscous regularization with a limited rate dependency. International Journal of Solids and Structures, 2001, 38 (18), pp.3127-3160. 10.1016/S0020-7683(00)00053-6 . hal-02093693

\section{HAL Id: hal-02093693 \\ https://hal.science/hal-02093693}

Submitted on 18 Apr 2019

HAL is a multi-disciplinary open access archive for the deposit and dissemination of scientific research documents, whether they are published or not. The documents may come from teaching and research institutions in France or abroad, or from public or private research centers.
L'archive ouverte pluridisciplinaire HAL, est destinée au dépôt et à la diffusion de documents scientifiques de niveau recherche, publiés ou non, émanant des établissements d'enseignement et de recherche français ou étrangers, des laboratoires publics ou privés. 


\title{
Interface debonding models: a viscous regularization with a limited rate dependency
}

\author{
J.L. Chaboche ${ }^{\mathrm{a}, *}$, F. Feyel ${ }^{\mathrm{a}}$, Y. Monerie ${ }^{\mathrm{b}}$ \\ a ONERA, DMSE/LCME, 29 avenue de la Division Leclerc, 92320 Châtillon, France \\ ${ }^{\mathrm{b}}$ CNRS, Laboratoire de Mécanique et d'Acoustique, 31 chemin Joseph-Aiguier, 13402 Marseille, France \\ Received 22 September 1998; in revised form 9 August 1999
}

\begin{abstract}
Interface models have been developed over the past 10 years for simulating various scales of composite debonding effects, such as decohesion between matrix and fibers and delamination in laminates, formulated with and without rate dependencies.

We give several examples of how sudden "solution jumps" can occur in rate-independent models like the Tvergaard depending on the geometry, interface behavior, and finite element discretization. This kind of instability disappears for the often-used viscous models, but the result is a high rate dependency that deviate from experimental trends.

A new kind of viscous regularization is proposed here, which applies to the Tvergaard debonding model or modified ones, and has a limited rate dependency. A few simple examples are given, including the delamination of a double cantilever beam, to show the capabilities of the technique proposed.
\end{abstract}

Keywords: Interface; Debonding; Delamination; Numerical modeling; Regularization techniques

\section{Introduction}

Interface damage mechanics (IDM) is a numerical tool, developed quite recently as part of continuum damage mechanics (CDM), with capabilities intermediate between CDM and fracture mechanics (FM). IDM - which stems independently from the works of Needleman (1987), Tvergaard (1990a,b), Allix (1987), Allix and Ladevèze $(1989,1992)$, Lemaître $(1992)$, and Frémond $(1985,1987)$ - is used to model the growth of a crack (or a debonding zone) at the interface between different components of the material. Compared with FM, which is based on numerical tools, it can generally be said that IDM models have three advantages: (i) smoothing the stress singularities at the crack tip, (ii) the continuity of the solutions in both space and time, and (iii) the easy adaptability with other non-linearities (plasticity, material damage, and so forth). 
IDM applications are mainly on two scales:

(1) Macroscale: This is where the strength of a laminated structure may be drastically reduced by the impact of an external solid or even under normal loading conditions in the proximity of geometrical defects (hole, reinforcement, or other). This delamination growth problem is a difficult one to solve in the framework of fracture mechanics, especially when we have other major non-linearities to consider, such as large displacements, damage, and/or plasticity in one or more layers and contact and friction in the delaminated area itself. Several works (Ousset and Roudolff, 1997) have shown the importance of contact and friction in delamination growth. One way of handling it would be to consider the intermediate layer between the plies as an individual component with its own constitutive equations. This was done by Allix (1987) and Allix and Ladevèze $(1989,1992)$ in the framework of a continuum damage mechanics approach, and by Lo and Allen (1994) using interface debonding models. Other approaches, based on Frémond's work (1987), use debonding models as a way of "regularizing" an FM analysis (Point and Sacco, 1996). Also, still on the macroscale, there is the possibility of modeling the adhesion and gluing.

(2) Microscale: This is the level of interaction between fiber and matrix. The role of these interfaces and interphases is clearly recognized. This is particularly evident in ceramic matrix composites, where the compromise between strength and ductility (i.e., dissipated energy) is essentially governed by the non-linear response of the interfaces, including progressive debonding and friction. Much research has been done in this area, developing comprehensive analytical approaches (among many others, see Budiansky et al. (1986), Marshall and Oliver (1987), Mc Cartney (1989), Hutchinson and Jensen (1990), Nairn (1992), and Evans and Zok (1994)). However, the limitations of the analytical models have also been demonstrated by finite element studies (Leroy et al., 1995; Guichet, 1998), so that there is still the need for more sophisticated interface debonding models and the associated numerical simulations.

The aim of the present paper is to review some of the numerical difficulties with interface debonding models, and to discuss various ways of overcoming these difficulties. After a brief review of a few of these models, and showing how they can be used for an "interphase material" (Section 2), we use a few examples (Section 3) to show how "solution jumps" may take place. (These solution jumps are discontinuities in the numerical solutions.) In Section 2, we study a number of viscous regularization processes that are used (explicitly or implicitly) in IDM models. But since the considerable rate dependency, resulting from these regularizations, should be reduced as much as possible, we propose (Section 5) a new viscous regularization technique that can be used with Tvergaard-type IDM models to curb this rate dependency. This modified model was used in the general-purpose ZéBuLoN 7 finite element code (Burlet and Cailletaud, 1986), and its capability is illustrated in a simple but significant structural example (Section 5.3).

\section{Interface debonding models}

\subsection{General features}

Interface damage mechanics models are used to describe the relationships between tractions (cohesion forces per unit area) and displacements across the interface during the debonding process. We denote the normal and tangential components of the traction vectors at the interface by $T_{\mathrm{n}}, T_{\mathrm{t}}$, respectively, and

$$
u_{\mathrm{n}}=\left[u_{\mathrm{n}}\right]=u_{\mathrm{n}}^{+}-u_{\mathrm{n}}^{-}, \quad u_{\mathrm{t}}=\left[u_{\mathrm{t}}\right]=u_{\mathrm{t}}^{+}-u_{\mathrm{t}}^{-}
$$

are the corresponding displacement differences across the interface. Positive $u_{\mathrm{n}}$ corresponds to increasing interface separation. We adopt the form of a cohesive model initially used by Needleman (1987) and generalized by Tvergaard (1990a) and Lissenden and Herakovich (1995), where the initial (elastic) cohesive response is gradually degraded using a decohesion indicator $d$ as damage parameter. In the same way as in 
CDM, we set this indicator initially at $d=0$ for the interface in perfect cohesion, and at $d=1$ for the completely de-bonded state.

In the Tvergaard model, the decohesion parameter, called $\lambda$, is given directly as the maximum value of the displacement norm, so that

$$
d=\lambda_{\max }=\max _{\tau<t} \sqrt{\left\langle v_{\mathrm{n}}\right\rangle^{2}+v_{\mathrm{t}}^{2}},
$$

where $v_{\mathrm{n}}=u_{\mathrm{n}} / \delta_{\mathrm{n}}$ and $v_{\mathrm{t}}=u_{\mathrm{t}} / \delta_{\mathrm{t}}$ are normalized displacements, and $\delta_{\mathrm{n}}$ and $\delta_{\mathrm{t}}$ are the normal and tangential displacements at complete separation for pure normal and pure tangential modes respectively (we assume that the tangential mode is isotropic). In relation (2), the Mc Cauley bracket \langle\rangle indicates specifically that a negative normal displacement plays no role in the decohesion (but is actually limited to very small values in the model).

In interface damage models, we can consider the non-linear relations between tractions and displacements (for positive $u_{\mathrm{n}}$ ) to be of the form

$$
\begin{aligned}
& T_{\mathrm{n}}=E F(d) v_{\mathrm{n}}, \\
& T_{\mathrm{t}}=G F(d) v_{\mathrm{t}},
\end{aligned}
$$

where $F(d)$ is a monotonically decreasing function of $d$, and $E$ and $G$ are the initial stiffnesses of the interface. In Tvergaard's version $F(d)$ is defined as $F(d)=(1-d)^{2}$, and $d$ as the maximum value encountered for the norm $\lambda$ of the displacement. This means that for purely normal separation, the maximum tensile stress is $\sigma_{\max }=4 E / 27\left(\tau_{\max }=4 G / 27\right.$ for pure tangential separation, and $\left.v_{\mathrm{n}}=0\right)$. Fig. 1 illustrates schematically the response under the two conditions.

In compressive mode (negative $v_{\mathrm{n}}$ and $T_{\mathrm{n}}$ ), the Tvergaard model describes the sliding processes (via Coulomb friction) after complete separation, as indicated in Fig. 1. Variants have been proposed by Chaboche et al., $(1997 \mathrm{a}, \mathrm{b})$ to obtain a monotonic response in the debonding process without increasing the stiffness after complete decohesion, which is one disadvantage in the initial version (as illustrated in Fig. 1). The friction properties in the model are now included throughout the whole debonding phase, with the tangential displacement decomposed into elastic and plastic components. (The equations for this modified
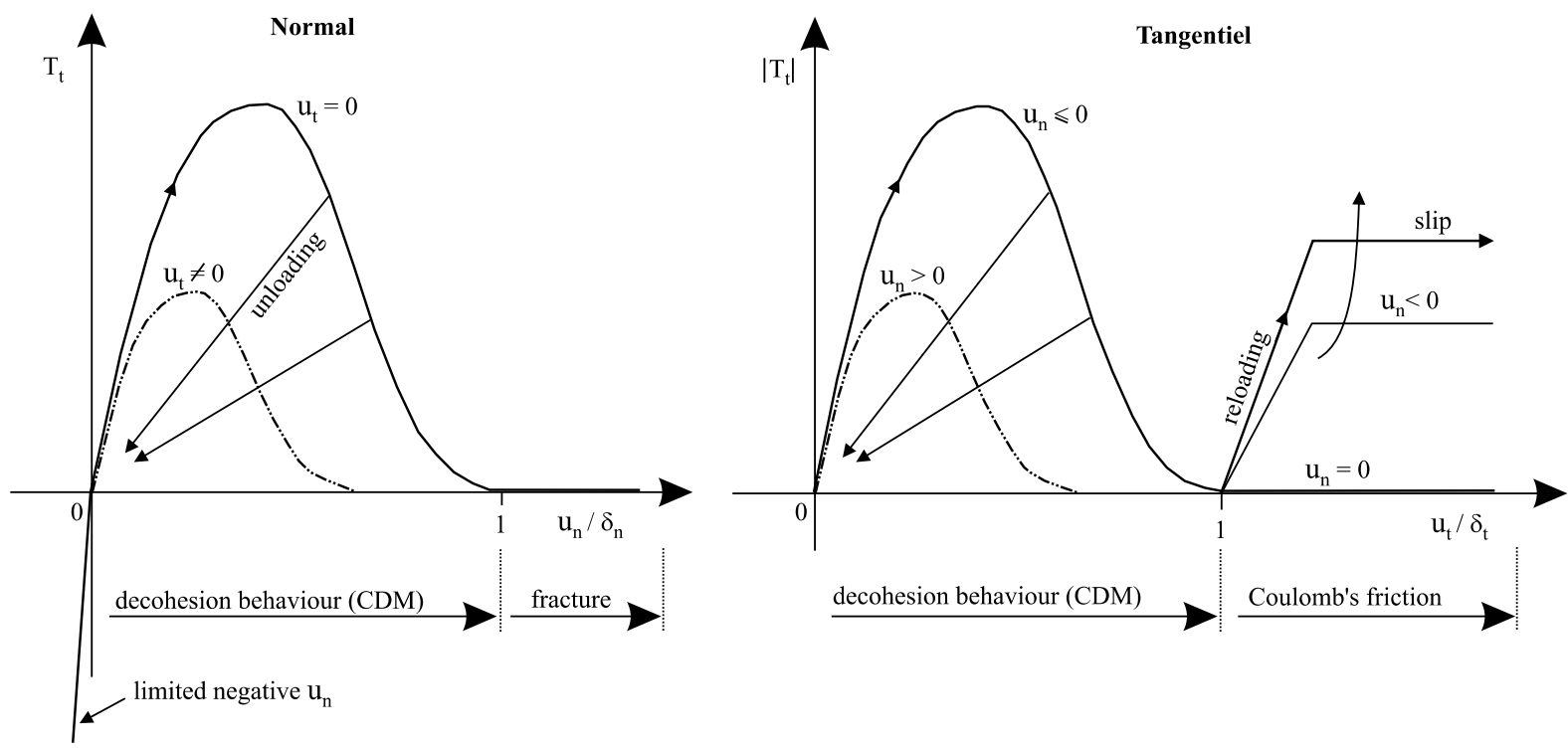

Fig. 1. The behavior of Tvergaard's interface debonding model: (a) under normal loading and (b) under tangential loading. 
model are recalled in Appendix A). For $u_{\mathrm{n}} \geqslant 0$, it is once again exactly like the Tvergaard model, so these aspects will not be discussed further here.

A number of slightly different interface debonding models have been proposed in the literature.

In the Allix and Ladevèze approach (Allix, 1987; Allix and Ladevèze, 1992), these models are deduced directly from the CDM models used for the bulk damage. Appendix B reviews the main lines of the formulation. Using the above notation, the decohesion effect is a linear function, $F(d)=1-d$. The damage process is written somewhat differently using the thermodynamic force associated with the damage variable. In the special case of the normal separation, which is sufficient for the purpose of comparing the models, this reduces to a linear relationship between damage and displacement, possibly with the introduction of a threshold $v_{0}=u_{0} / \delta$, so that

$$
d=\frac{v-v_{0}}{1-v_{0}} .
$$

The model developed by Cangémi et al. (1995) uses strict inequalities (penetration is strictly prevented), following Frémond's approach $(1985,1987)$. The main lines of the model are given in Appendix B. Contrary to the Tvergaard model, this one uses a damage threshold. For tensile-like loading below the threshold $\left(u_{\mathrm{n}}>0\right)$, it returns an elastic response. Above the threshold, debonding takes place and the elastic stiffness is gradually reduced. The main differences are the rate-dependency of the damage process (discussed below in Section 4) and the exponential character of the traction/displacement response, as illustrated in Fig. 25.

The rate-independent versions of each of the three models can be adjusted either by considering the maximum stress or energy dissipated before complete separation or the initial stiffness or displacement at complete separation. To do this, only two of these parameters have to be adjusted, since the others are involved only approximately, as shown in Appendix B. The corresponding comparisons are given in Fig. 25.

\subsection{Interface as interphase model limit}

There is a problem of theory when developing interface models. They may be used either to describe the behavior of an interphase material of given thickness, or the separation between two parts of a solid, like a crack with no thickness (i.e. the limiting case with a zero thickness). This problem has been addressed directly a number of times in the past, e.g. by Suquet (1987) and Michel et al. (1994), but it is in any case a problem underlying many mathematical models.

Starting with the elastic, plastic and damage constitutive equations for an "interphase", written in terms of bulk strain, stress, and damage, it is easy to formulate the corresponding "interface" constitutive equations.

Assuming that the thickness $h$ is small and that the strains throughout this thickness are uniform, we have (Suquet, 1987)

$$
\varepsilon=\frac{1}{h}(\mathbf{u} \otimes \mathbf{n})_{\mathrm{s}}
$$

in which $\mathbf{n}$ is the unit normal to the interface. Assuming that the strain and elastic-plastic partitioning is small, we can also say that

$$
\varepsilon_{\mathrm{e}}=\frac{1}{h}\left(\mathbf{u}^{\mathrm{e}} \otimes \mathbf{n}\right)_{\mathrm{s}}, \quad \varepsilon_{\mathrm{p}}=\frac{1}{h}\left(\mathbf{u}^{\mathrm{p}} \otimes \mathbf{n}\right)_{\mathrm{s}} .
$$

Let us postulate that the elastic behavior of the interphase is

$$
\boldsymbol{\sigma}=\mathbf{C}(d): \varepsilon_{\mathrm{e}},
$$

where $\mathbf{C}$ is a fourth-rank tensor that depends on the interphase damage $d$, which is also assumed to be constant throughout the thickness here. We now have the tensile loads at the boundary between the "interphase" and the matrix (resp. fiber) with 


$$
\mathbf{T}=\boldsymbol{\sigma} \cdot \mathbf{n}=\frac{1}{h} \mathbf{n} \cdot \mathbf{C}(d):\left(\mathbf{u}^{\mathrm{e}} \otimes \mathbf{n}\right)_{\mathrm{s}}
$$

that can be expressed (Michel et al., 1994) as

$$
\mathbf{T}=\mathbf{K}(d) \cdot \mathbf{u}^{\mathrm{e}}, \quad \mathbf{K}(d)=\frac{1}{h} \mathbf{n} \cdot \mathbf{C}(d) \cdot \mathbf{n} .
$$

By comparison with Eqs. (3) and (4), we immediately obtain (in the 2D case):

$$
K_{\mathrm{nn}}=\frac{E}{\delta_{\mathrm{n}}} F(d)=\frac{1}{h} C_{\mathrm{nnnn}}(d), \quad K_{\mathrm{tt}}=\frac{G}{\delta_{\mathrm{t}}} F(d)=\frac{1}{h} C_{\mathrm{ntnt}}(d), \quad K_{\mathrm{nt}}=0 .
$$

We now simplify the analysis by postulating the simplest scalar dependency for the interphase damage:

$$
\mathbf{C}(d)=F(d) \mathbf{C}^{0} .
$$

With this, Eq. (11) will then degenerate into

$$
E=\frac{\delta_{\mathrm{n}}}{h} C_{\mathrm{nnnn}}^{0}, \quad G=\frac{\delta_{\mathrm{t}}}{h} C_{\mathrm{nnnt}}^{0} .
$$

If we want to achieve a finite stiffness $(E$ and $G$ ) for the interface model (with the corresponding maximum for finite tensile loads $T_{\mathrm{n}_{\max }}, T_{\mathrm{t}_{\max }}$ ) and if we further consider that the interface is of zero thickness, i.e. the limit as $h \rightarrow 0$, we have to assume that the compliance for the corresponding "interphase" elastic behavior is singular with linear dependency on the thickness

$$
\mathbf{C}^{0}=h \overline{\mathbf{C}}
$$

Although this assumption is used sometimes in certain applications, it does not seem to be realistic for the continuum properties of the material. So, we would rather adopt one of the following possibilities:

(1) The interphase to be modeled really is of given finite thickness. This way, the interface model is found by integrating through the thickness, and its constitutive law is not intrinsic, but rather depends on the thickness, as in Eq. (13). This is true in many applications for composite systems, where the matrix is separated from the fiber by one or more interphase materials. This is also true for delamination problems in laminates, where a thickness of pure matrix can be identified between two adjacent plies.

(2) The other possibility is to consider that the interface is not a given interphase thickness, but rather describes the decohesion in terms of crack growth. In this case, the interface model should not be thought of in terms of the limit problem as before (though an analogy is still possible), but it can be postulated independently of any bulk interphase properties in which case the interface debonding model is now viewed as a "spatial regularization" of the fracture mechanics model. This is part of the approach proposed by Frémond (1985), and can also be found in the regularization methods used by Point and Sacco (1996), for example. From the Tvergaard interface model, for instance, we can construct its corresponding fracture mechanics counterpart by decreasing the displacement at complete separation $\delta$, indefinitely, but maintaining a constant fracture energy $\left(G_{\mathrm{I}_{c}}=(1 / 12) E \delta_{\mathrm{n}}, G_{\mathrm{II}_{c}}=(1 / 12) G \delta_{\mathrm{t}}\right)$. If the separation is normal, for instance, we again have $E=12 G_{\mathrm{I}_{c}} / \delta_{\mathrm{n}}$, but with $\delta_{\mathrm{n}} \rightarrow 0$ now ( $\delta_{\mathrm{n}}$ is no longer the interface thickness, but the displacement at separation). Fig. 2 illustrates this analysis with the case of (elastic) fracture mechanics at the limit involving infinite stress and zero opening, i.e. the conditions of a sharp crack tip. The area under each curve in Fig. 2(b) represents the same fracture energy. It should be remembered that the displacement is zero only at the crack tip but that the opening and therefore the displacement is non-zero for any points that are already separated.

Let us also point out the transition between the interphase plasticity and the interface slip property (just the tangential plastic displacement $u_{\mathrm{t}}^{\mathrm{p}}$ ) in the modified model of Appendix A. For the interphase plasticity, we have to adopt the Drucker-Prager yield criterion (which depends linearly on the hydrostatic stress) and 


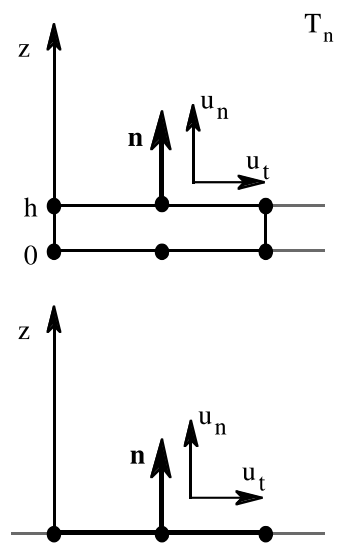

(a)

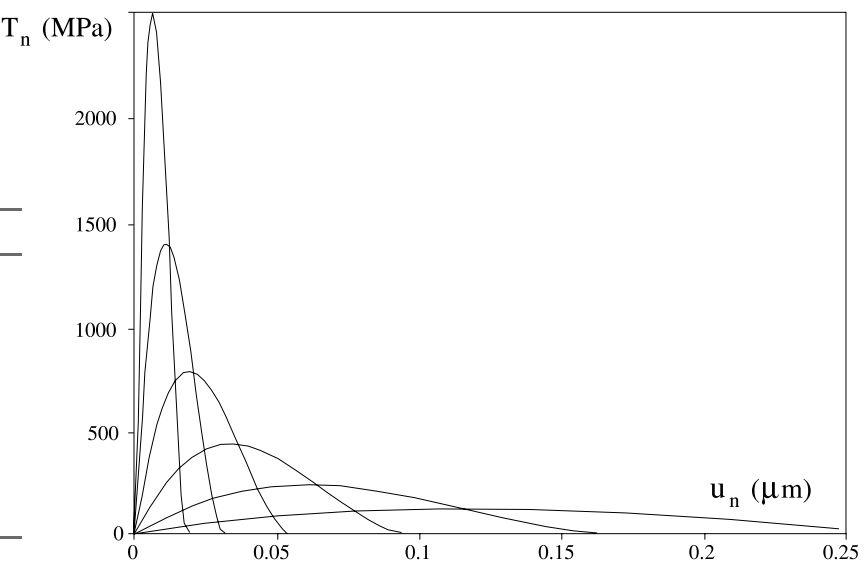

(b)

Fig. 2. (a) The correspondence between a "volumic interphase" of thickness $h$ and an "interface" and (b) the interface model as a regularization of fracture mechanics singularity.

assume that the change in volume is negligible (non-associative flow rule), in which case, due to the confinement of the small interphase thickness between more rigid materials, it is consistent to assume that the normal plastic strain is zero, i.e. $\dot{\varepsilon}_{\mathrm{nn}}^{\mathrm{p}}=0$, so that we now have $u_{\mathrm{t}}^{\mathrm{p}}=0$.

\section{3. "Solution jump" problem}

Problems arise when we try to simulate the deterioration processes and their corresponding softening effects in the structural components. In the normal CDM framework, where damage is described as a bulk state variable (3D formulation), the "localization" (mesh dependency), non-uniqueness, and bifurcations will degrade the simulation of these processes as they lead into the local failure (crack initiation and growth through a complete local deterioration of the material strength and stiffness). Over the past 10 years, a great deal of research has been done to solve such problems, by way of localization limiters or using the concepts of generalized continuum mechanics (Bazant and Pijaudier-Cabot, 1988; Belytschko and Lasry, 1988; Saanouni et al., 1989; Benallal, 1991; Bigoni and Hueckel, 1991; de Borst, 1991; Murakami et al., 1993).

The localization problem is not present in the present context of interface damage mechanics, because the interface is already a localized model. Moreover, this bifurcation point is special: it induces a loss of stability, because the second-order work is zero (Bazant and Cedolin, 1991), but the uniqueness is not lost. The simple example below shows how this instability occurs in the rate-independent formulation, and induces a response discontinuity that we call a "solution jump". The example also illustrates the "snapback" character of instability.

\subsection{Simple uniaxial example}

Let us assume the simplest 2D structure. This will consist of an interface element loaded normally through an elastic plate element of given geometry and stiffness. Fig. 3(a) illustrates the example in terms of both the finite element geometry and of its behavior in series. The same example also holds for pure shear conditions (Monerie et al., 1998). Let us consider the total displacement control at the position BB'. Obviously, the total displacement $U$ is the sum of the interface separation displacement $u$ and the elongation of elastic adjacent part $\mathrm{AA}^{\prime} \mathrm{B}^{\prime} \mathrm{B}$ 

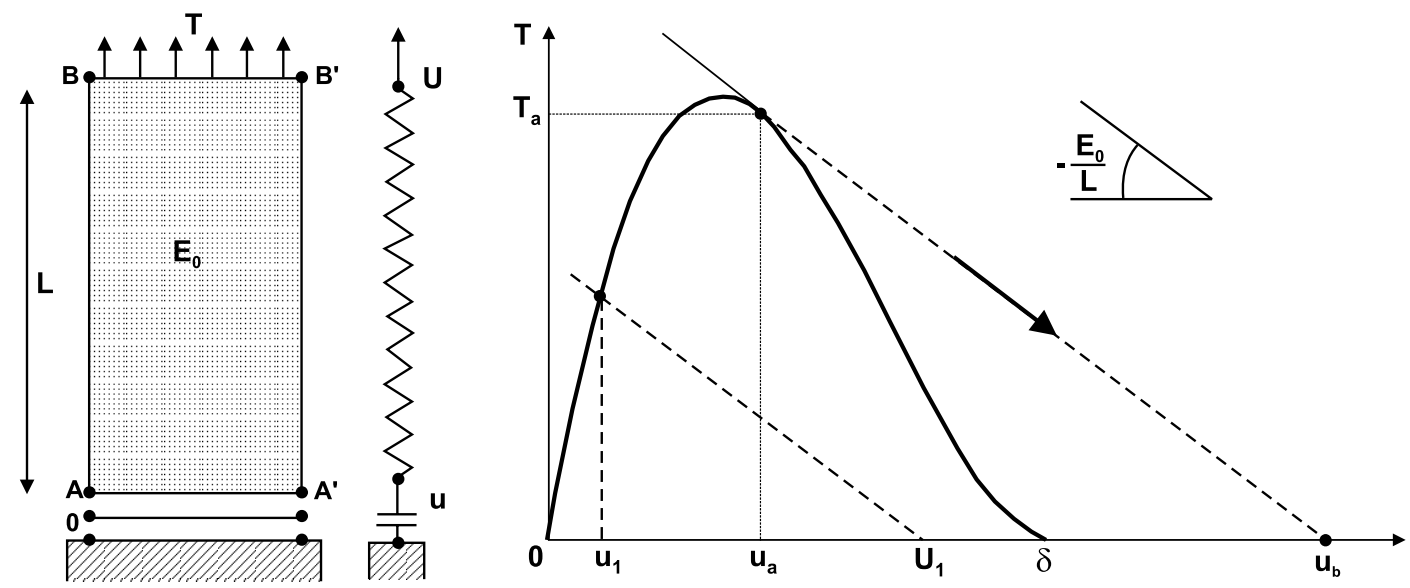

Fig. 3. The simple uniaxial configuration that demonstrates the possibility for "solution jumps": (a) the interface and plate elements in series and (b) the local interface response, loaded via the global displacement control $U$.

$$
U=u+\frac{L}{E_{0}} T
$$

where $T$ is the stress applied at the end $\mathrm{BB}^{\prime}, E_{0}$, Young's modulus and $L$, the length of the elastic part $\mathrm{AA}^{\prime} \mathrm{B}^{\prime} \mathrm{B}$.

In the interface, behavior is described through a Tvergaard debonding model, we have

$$
T=E F\left(\frac{u}{\delta}\right) \frac{u}{\delta}
$$

where $u$ is assumed for convenience to vary between 0 (initial condition) and $\delta$ at complete separation and with $F(0)=1, F(1)=0$. In the local coordinates $(T, u)$, Fig. 3(b) shows the local (interface) response together with the straight lines corresponding to the global displacement control, given by Eq. (15). Obviously, when the control $U$ is increased, after the maximum of the local response (16), either of two situations may occur, depending on the stiffness $k=E_{0} / L$ of the adjacent elastic structural part:

1. The stiffness $k$ is large enough to maintain the possible intersection between the straight line (15) and the local response (16), in which case the response is always stable.

2. The stiffness is less than the maximum tangent stiffness (negative slope) in the local interface. In this situation, as control $U$ increases, we obviously expect to find a jump in the solution (from $u_{a}$ to $u_{b}$ in the figure 3 (b), from $T_{a}$ to $T=0$ in terms of the load).

With the Tvergaard model, the maximum slope of the softening curve is at $u=2 \delta / 3$, with a value $-E / 3 \delta$. In that case, the two situations above can be written respectively as

$$
\frac{E_{0}}{L} \geqslant \frac{1}{3} \frac{E}{\delta} \rightarrow \text { stable, } \quad \frac{E_{0}}{L}<\frac{1}{3} \frac{E}{\delta} \rightarrow \text { jump. }
$$

This "solution jump" entrains no loss of uniqueness, because instantaneous failure is obviously the exact solution of the present elementary mechanical problem. Moreover, if we consider the global control problem, we do have the snap-back situation: no increase in global displacement is possible if the local response is followed continuously. The global system will jump directly from $a$ to $b$, though following the local behavior would require a reduction of the global displacement. In other words, when the local 
behavior begins to soften (negative slope), the elastic energy stored in the surrounding material is released rapidly by the interface as its damage increases, and can be sufficient to break it instantaneously.

It will be shown in the following that this simple example becomes generic for the above numerical instabilities in a number of situations. Let us note a recent development of this example (Delaplace et al., 1998), where the elementary behavior is decomposed in terms of a hierarchy of discrete microelements. However, it confirms the unstable character of the global response.

\subsection{Examples of interface debonding simulations}

In a previous paper (Chaboche et al., 1997a,b), the Tvergaard interface model was used to describe the interface crack growth (mode II) along a fiber bridging a matrix crack in a unidirectional axisymmetric composite cell, loaded in axial tension. The cited study will not be reviewed here, but the aspects related to solution jumps will.

In the rate-independent solution (Tvergaard model), the interface debonding begins at the intersection of the matrix crack and fiber interface (there is, in fact, an elastic singularity). Fig. 4 illustrates the variation of the shear stress distribution along the fiber interface as the global elongation of the composite cell gradually increases. The shear stress is initially maximum at the matrix crack tip (left of the figure, steps 1-5), and then decreases gradually there, as the maximum position moves to the right. Before reaching zero at the crack tip (complete local separation at the first interface node), there is a sudden jump on the right (between steps 8 and 9) with instantaneous failure at several nodes. The remainder is stable, with a continuous growth of the interface damage to the right of the figure (the end of the composite cell).

Conversely, it is worth noting that an analogous rate-dependent interphase model would deliver similar shear stress distributions, but with no solution jump (compare Fig. 4(a) and (b)). In that case, the two damage models were both adjusted for the same rate domain as in the rate-independent solution (Chaboche et al., 1997a).

The solution jump for the rate-independent model was also observed for the delamination simulation in a double cantilever beam, at least at the onset of crack growth initiation. It might be mentioned that the "solution jump" is also related to the mesh size (as will be discussed below). Tvergaard himself showed this dependency in a few numerical examples, without discussing them at length (Tvergaard, 1990b). Certain

(a)

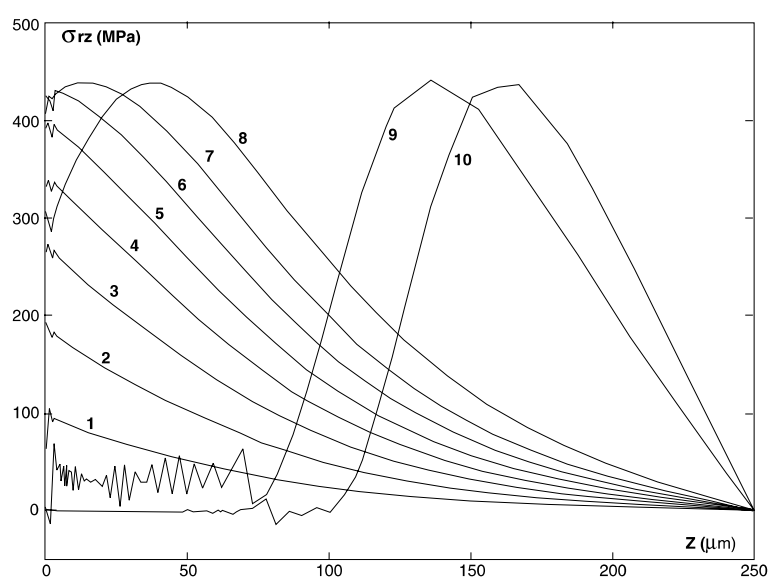

(b)

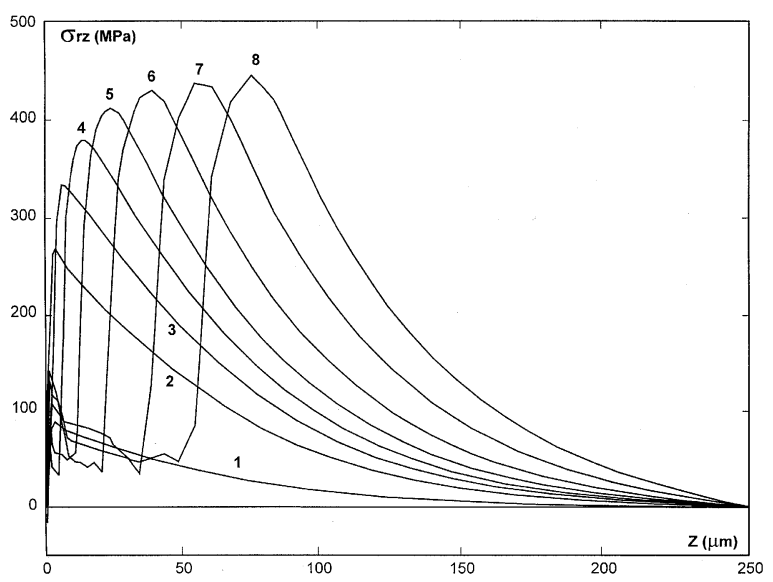

Fig. 4. The shear stress fields at the interface between fiber and matrix during the "pull-out" from a perpendicular matrix crack: (a) the rate independent Tvergaard interface model and (b) the rate dependent CDM interphase model. 
workers who have used this and other similar models (Lo and Allen, 1994; Lissenden and Herakovich, 1995; Lissenden, 1996) make no mention of this problem, though it probably did exist (depending on the mesh size or the integration procedure).

It is worth noting that the situation in which the solution jump will arise is not an easy one to control numerically. Roughly speaking, this is because two solutions exist at the same time, and this can disturb the performance of Newton-like implicit schemes. If we refine the time step (by constant stepping or automatic time stepping), we make the problem harder rather than solving it, as the numerical scheme comes closer to running into this jump situation. Increasing the time step, on the other hand, will deliver a solution with a jump, but we can never be sure if the solution beyond the jump is actually valid, especially when several interface nodes are broken simultaneously.

\subsection{Mesh dependency}

The simple generic example of Section 3.1 has demonstrated the possibility of a solution jump in a very academic situation. For a given interface (given values of $E$ and $\delta$ ) there exist a size $L$ of the adjacent structure (of modulus $E_{0}$ ) below which the solution jump does not occur. Conversely, for a given structure (or mesh size), and a given initial stiffness of the interface, there is a displacement $\delta$ at complete separation, above which the solution jumps no longer occur. In practice, however, we cannot choose any large values for $\delta$ that would lead to unrealistic values of the interface toughness.

The bending example treated here is designed to generalize these results for a structure used in simulating a micro-indentation experiment (another such generalization is proposed in Monerie et al. (1998)), in which the debonding of the fiber-matrix interface occurs in mode II. It is also intended to show that, given certain interface characteristics, the "solution jump" can be eliminated by refining the mesh of the adjacent structure sufficiently.

We consider a beam subjected to pure bending (at least to start with). The beam is in two parts, separated by an interface (Fig. 5). The right part is a $1 \times 1 \mathrm{~mm}^{2}$ square plate (plane strain). It is meshed with square finite elements (eight nodes). It is assumed elastic and isotropic, with $E_{0}=70.000 \mathrm{MPa}$ and $v=0.2$. The left part is assumed rigid. The interface model between the two parts is modeled with $E=G=675$ $\operatorname{MPa}\left(\sigma_{\max }=100 \mathrm{MPa}\right)$ and $\delta_{\mathrm{n}}=\delta_{\mathrm{t}}=1 \mu \mathrm{m}$. The axial displacement is controlled as linear at the right end and increases linearly in time. The problem is treated in small displacements. Successive mesh refinements have been studied, from $3 \times 3$ elements to $40 \times 40$.

Under these conditions, the interface begins to deteriorate in pure mode I, on the upper side of the beam. Fig. 6 shows the local stress-displacement curves of the upper interface node (we should point out that the local interface properties are not reproduced exactly, because the stress values are obtained by extrapolating the Gauss point values in the first element).

However, the "solution jump" after the peak is clearly observed for the larger mesh sizes. We note that a second or a third peak can occur before the complete failure, due to the deterioration of the next nodes. More interesting yet is the disappearance of the solution jump for smaller mesh sizes and the correct convergence $(20 \times 20-40 \times 40)$.

The upper side of the beam is initially under a near-uniform tensile axial stress (along the $x$ axis) that is analogous to the case of the simplest uniaxial example of Section 3.1. However, for finer meshes, the elastic energy present in the region of this upper side is redistributed sufficiently when the first interface node soften (see the shear stress field in Fig. 5).

Due to this redistribution, which is allowable for sufficiently fine meshes, the solution jump no longer exists. Fig. 7 shows the local interface displacement (at the upper node) just before and just after the solution jump, as a function of the first element local stiffness $E_{0} / h$ ( $h$ is the size of the element). These values are below and above $2 / 3$, which is the theoretical position for the largest negative tangential modulus of the 


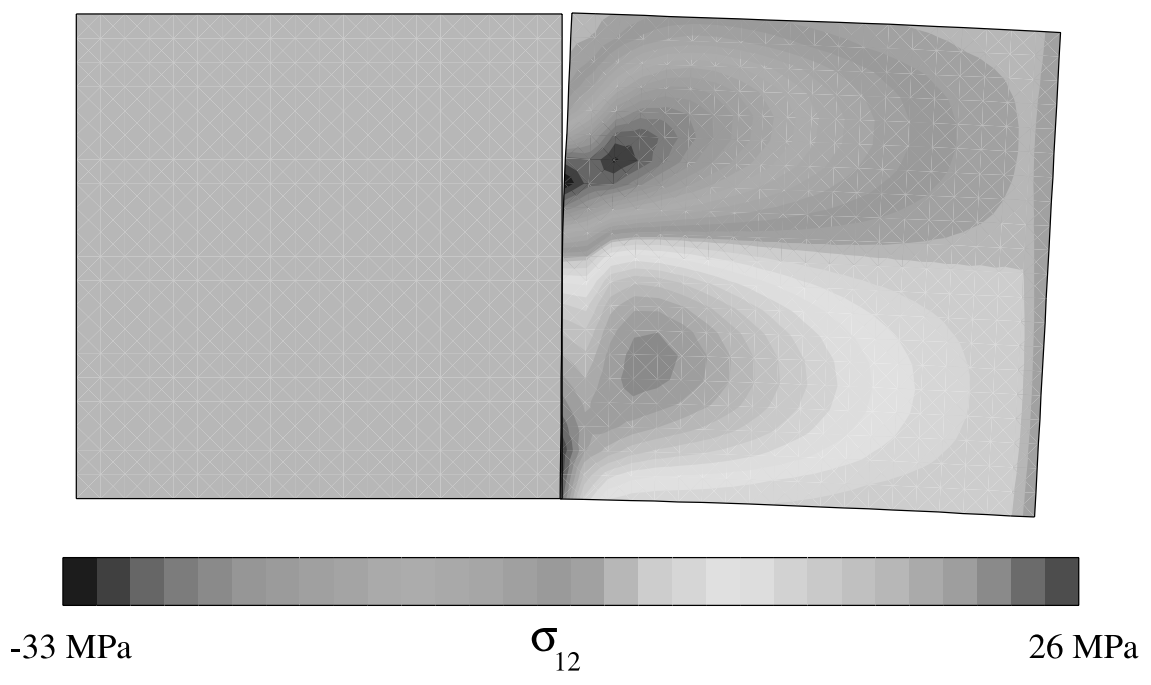

Fig. 5. The shear stress field after the sudden crack growth (jump) in the bending example. The right part is modeled by $20 \times 20$ square elements. The left part is rigid, shear stress lines correspond to $2 \mathrm{MPa}$ intervals, between $-33 \mathrm{MPa}$ (upper region) and $+26 \mathrm{MPa}$ (lower region).

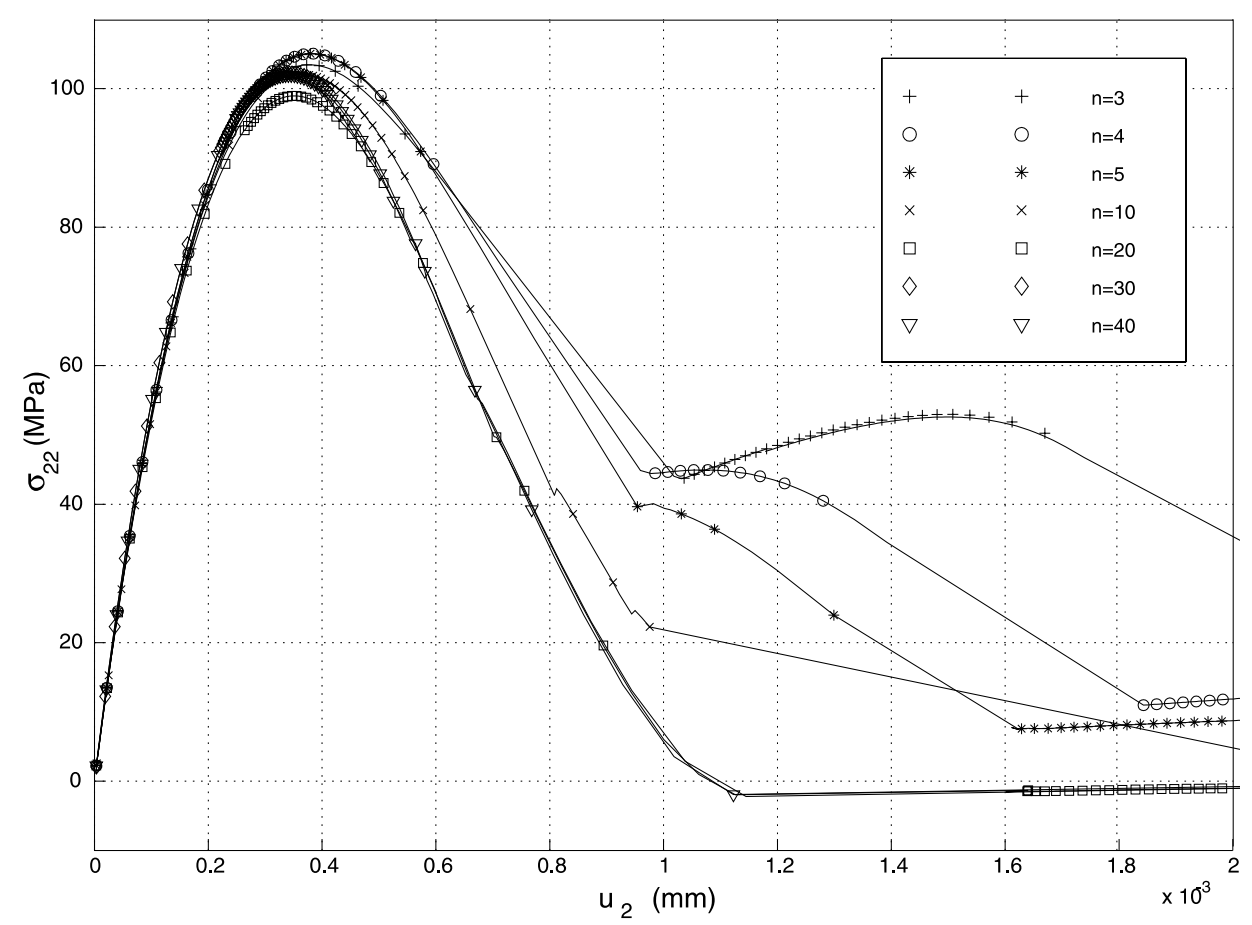

Fig. 6. Local stress-interface displacement at the upper node for various mesh sizes from $3 \times 3$ to $40 \times 40$.

interface (for larger numbers of elements, there seems to be no way of distinguishing between the normal loading increment and a solution jump). 


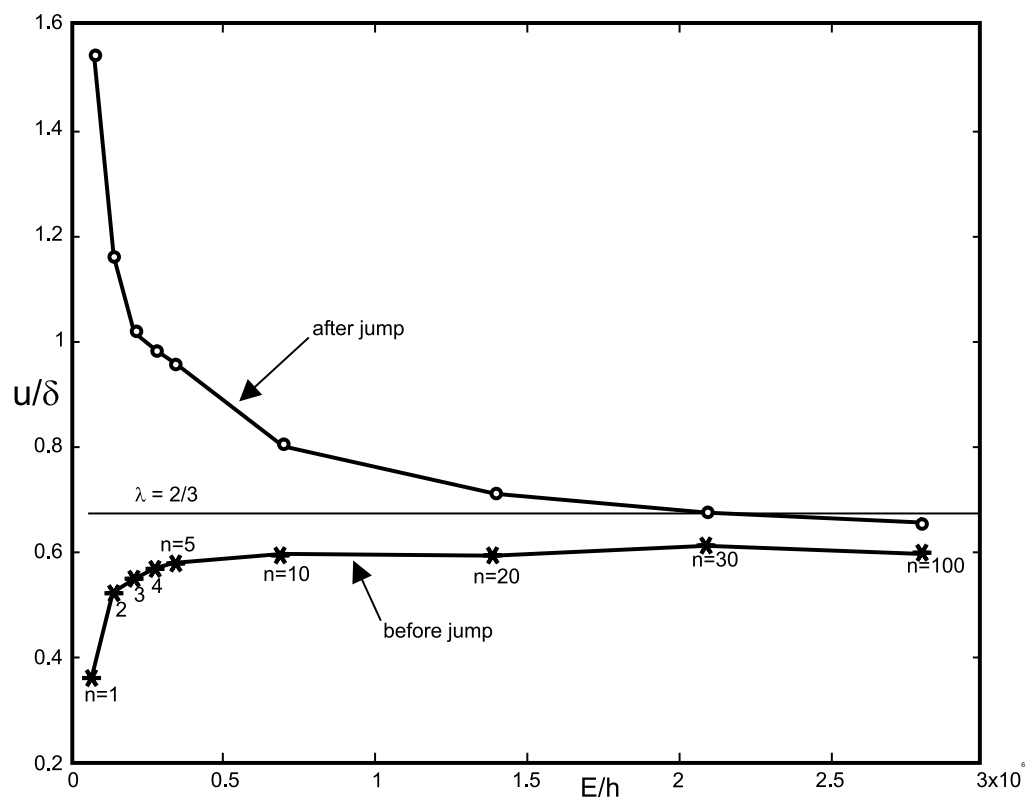

Fig. 7. Local interface displacement just before and just after the solution jump, as a function of the first element local stiffness $E / h$ $(h=$ size of the element).

On the basis of this example, we can make the following remarks:

- This structure is very simple, as it is the simplest one that can be found beyond the elementary uniaxial case.

- Although the initial uniformity of the stress field runs along the upper side, the elastic energy will be redistributed well as long as the mesh is fine enough and, if it is, there is no longer any solution jump.

- This confirms that the jump problem is mesh dependent and that, for a given set of interface material parameters $(E$ and $\delta)$, it can be eliminated by using mesh refinements near the interface.

\subsection{Possible methods to overcome the jump problem}

From the various examples treated, we may consider several procedures in order to eliminate the jump or to control the solution:

1. As we have just shown, we can refine the mesh in the region of interest. This has already been shown in Tvergaard's simulations (1990b). However, for realistic interface parameters, i.e. low values for $\delta$, the mesh sizes have to be extremely small, which is not acceptable for computational efficiency.

2. Special control procedures could be developed to detect the proximity of a solution jump, without recourse to Newton's method, to prepare for this jump, and then to set it explicitly for the loading instant just after the jump. Such a procedure might be efficient, but it would be too complicated for general-purpose use in a finite element code.

3. We "control" the solution locally. Once we have detected the node that will "jump" next, replace the external control (from the external displacement or load) to the local interface displacement. It will force the local solution to follow the local interface response curve, inducing a "snap-back" of the global one. 
The whole sequence of states will then be intermediate fictitious equilibrium states holding to the stable constitutive equations, but not to the global controlled loading. At the end, when the control returns to its set value, we check both the equilibrium, the boundary conditions, and the constitutive properties.

4. Perform a dynamic analysis instead of a quasi-static one. In this case, the elastic energy, when suddenly released, can be transformed into kinetic energy of certain parts.

5. Use a "non-local" formulation for the interface model, coupling the deterioration state of one point (node) with the one of neighboring interface points. As is true for the non-local damage solution (Bazant and Pijaudier-Cabot, 1988; Saanouni et al., 1989; Murakami et al, 1993; Baaser and Gross, 1998) in the volume damage growth, a non-local formulation like this could smoothen the solution and reduce or eliminate the jump. This is not addressed in the present article, though.

6. Use viscous regularization techniques to delay the sudden release of energy, and recover the continuity of the solution at each point in time. As in the previous case, it then modifies the constitutive equations of the interface itself. These techniques will be discussed in the following two sections.

\section{Analysis of several viscous regularizations}

The principle of this was given by Duvaut and Lions (1972) in the framework of plasticity/viscoplasticity. The rate-independent solution (or inviscid solution), in fact the yield surface equation, is used to build up a viscous potential that yields the same solution for infinitely low rate conditions. We will be looking here at three such kinds of regularization processes as they apply in the CDM framework, especially for interface modeling.

The Allix and Ladevèze (1989) method uses the rate-independent formulation through the relation that does exist between the damage parameter and its associated thermodynamic force $y$. In other theories, this relation is used to define the surface within the $y$ space where the damage is not growing. In the simple case of one single variable, this relation is expressed:

$$
d=\omega(y) \text { if } d<1, \quad d=1 \quad \text { otherwise, }
$$

where $y$ is defined by $y_{\mathrm{t}}=\max _{\tau<t} y_{\tau}$. The material function $\omega$ is often taken to be bi-linear in the displacement:

$$
\omega(y)=\left\langle\frac{\sqrt{y}-\sqrt{y_{0}}}{\sqrt{y_{c}}-\sqrt{y_{0}}}\right\rangle=\left\langle\frac{u-u_{0}}{u_{c}-u_{0}}\right\rangle .
$$

This equation appears when considering the simple case with only one displacement component, which is sufficient here (assuming the normal case) as long as $y=(1 / 2) E u^{2} / u_{c} . y_{c}$ and $u_{c}$ are values of $y$ and $u$ at complete separation and $y_{0}$ respectively, $u_{0}$, their values at the onset of the initial damage growth. The viscous regularization is obtained through a delay effect (Ladevèze, 1992, 1995; Allix and Ladevèze, 1996), with Eq. (17) replaced by

$$
\dot{d}=\frac{\eta}{a}[1-\exp (-a\langle\omega(y)-d\rangle)]
$$

if $d<1$, otherwise $\dot{d}=0$. There is a certain delay defined by a characteristic time $a / \eta$. Examples of the regularization capabilities of this technique have been presented by Ladevèze (1995). The rate dependency of this model is quite low in the low rate region. However, in the higher rate domain, it is very high, as can be seen in the simulations of Appendix $\mathrm{C}$, and in the figure, that relates the maximum traction to the rate $\dot{u}$, for a given value of $\eta / a$. 
Cangémi et al. (1995) use an interface debonding model constructed in a thermodynamic context that relates the damage rate to the difference between the damage thermodynamic force and a threshold energy $w$

$$
\dot{d}=\frac{1}{b}\langle y-w\rangle=\frac{1}{b}\left\langle c(1-d) u^{2}-w\right\rangle .
$$

In this model also, the rate dependency is very high, at least in the high-rate regime, far from the inviscid solution which is given by equality $c(1-d) u^{2}=w$ and Eq. (C.5). Appendix C shows a few simulations for constant rates $\dot{u}$. In the high-rate region, far from the inviscid solution, we can neglect the threshold $w$ and the corresponding closed form solution shows a $1 / 3$ power dependency between the rate $\dot{u}$ and $T_{\max }$ (Eq. (C.14) in, Appendix C), as shown in Fig. 8.

With a creep damage equation, Chaboche et al. (1997a) have simulated interphase behavior, as shown in Section 3.2. The corresponding interface model can be written as

$$
\begin{aligned}
& \dot{T}=E(1-d) \frac{u}{\delta}, \\
& \dot{d}=\frac{1}{\tau_{0}}\left(\frac{T}{A}\right)^{r}(1-d)^{-s},
\end{aligned}
$$

where Eq. (22) obeys Rabotnov's creep damage equation. As shown in Appendix C, this model can be integrated in closed form for a given constant-rate displacement control $u=\dot{u} t$. Then, the condition for the maximum traction is given by Eq. (C.19) from the solution of $\partial T / \partial u=0$. In the application by Chaboche et al. (1997a), we have used the particular parameter choice $s=r=2$, so that Eq. (C.19) reduces to Eq. (C.20). It shows exactly the same rate dependency as the previous model in the high-rate process. The corresponding prediction is a straight line in Fig. 8.

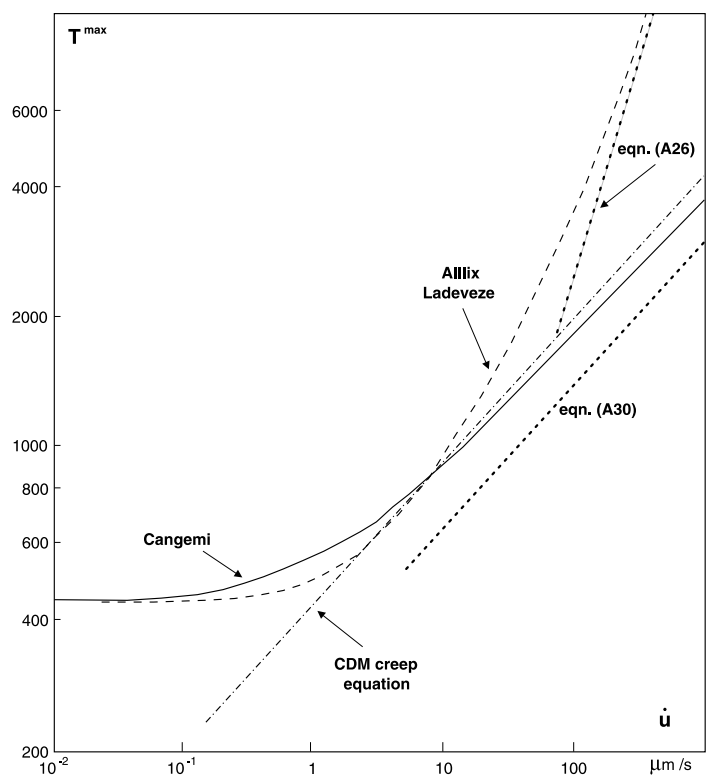

Fig. 8. Relation between the maximum traction and the controlled local displacement rate for three viscous interface models. Dotted lines represent approximate asymptotic solutions. 

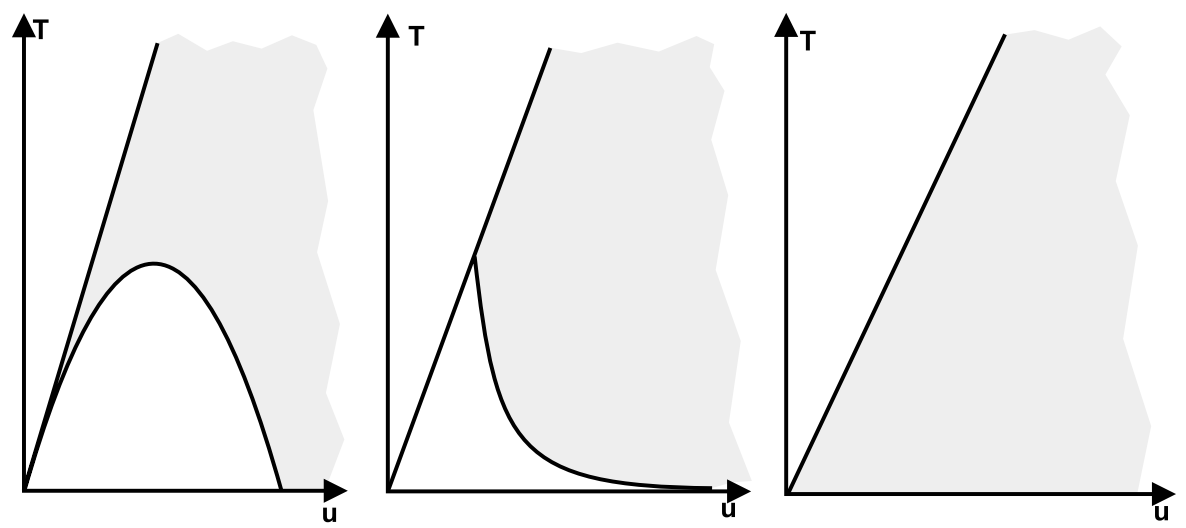

Fig. 9. Schematics of the acceptable subspaces for the traction/displacement solution in the three viscous interface models: (a) AllixLadevèze, (b) Cangémi et al. and (c) CDM creep equation.

As expected, the three models above contain a great rate dependency, at least in the high-rate process, as shown by Fig. 8. Compared to the rate independent case, where the local interface response is given by a unique tensile-displacement curve, these models exhibit a very large subspace for acceptable solutions (on the $T-u$ plane). Fig. 9 schematically illustrates this property for the three models. In the two first cases, the subspace of acceptable solutions, represented by the gray area, lies between the inviscid solution (rateindependent solution) and the undamaged elastic line. In the third case, the whole subspace below the initial elastic line is acceptable.

Obviously, such a property has a highly regularizing effect, and the problem of "solution jumps" will never be encountered using these interface models. However, they do correspond to a very different behavior of the material, compared with the rate independent case. In practice, the energy dissipated by the viscosity can at times be much larger than that corresponding to the pure decohesion energy. Then, the identification of the interface models from fracture toughness values requires at least a careful examination of the corresponding rate problem (and in fact numerical simulations in some structural cases). Moreover, even if the energy dissipation is correctly identified for a given rate domain, the models will definitely show a significant rate dependency, of the order of what was analyzed above, for the local models as well as for a structural application. Such behavior can be very far from the effects actually observed, especially for brittle materials like ceramic matrix composites, even if certain rate dependency or creep effects have been indicated (Cangémi, 1997). This is why we search for a regularization technique that minimizes the rate dependency, as discussed in the following section.

\section{Development of regularization with limited rate dependency}

The above rate-dependent models can be modified by changing the parameters. For example, in the second and third cases, we can introduce other values for the parameters in order to reduce the $(1 / 3)$ exponent that applies to the rate in Eqs. (C.14) and (C.20). However, this does not change the fact that the upper limit of the subspace for acceptable solutions in the $T-u$ space is given by the undamaged elastic line. Fig. 9 shows this acceptability domain for the three viscous models considered in Section 4, as the region between the inviscid solution and the undamaged elastic response.

Because the "solution jumps" are induced by the negative slope in the softening part, we are looking for regularization processes in which the acceptable subspace for solutions would be defined by the inviscid 


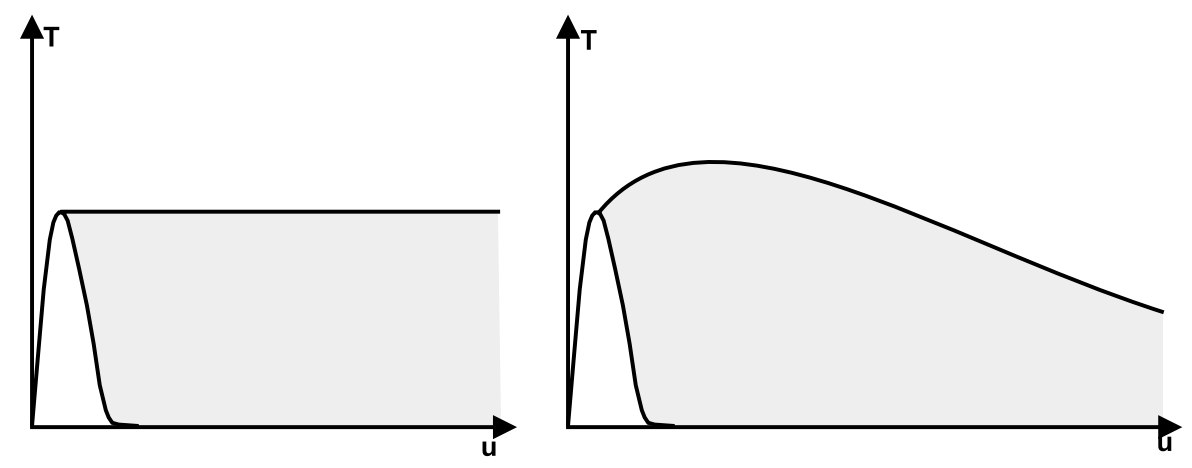

Fig. 10. Schematics of the acceptable subspaces for the traction/displacement solution in the Tvergaard model regularized with a limited rate dependency: (a) the homothetical rule and (b) the logarithmic rule.

solution (lower limit) when $\dot{u} \rightarrow 0$, and the line $T=T_{\max }$ for the upper limit as $\dot{u} \rightarrow \infty$. Fig. 10(a) illustrates the property we are looking for. This is expected to regularize the jump problem completely, but while introducing the minimum amount of viscous dissipation at the same time.

Two versions are evaluated below, each based on the rate-independent version of the Tvergaard model. We denote for convenience $\lambda$, the norm of the normalized displacement

$$
\dot{\lambda}=\sqrt{\left\langle\frac{u_{\mathrm{n}}}{\delta_{\mathrm{n}}}\right\rangle^{2}+\left(\frac{u_{\mathrm{t}}}{\delta_{\mathrm{t}}}\right)^{2}} .
$$

The value of $\lambda$ corresponding to the maximum tension $T=\|\mathbf{T}\|=E \sqrt{\left(T_{\mathrm{n}} / E\right)^{2}+\left(T_{\mathrm{t}} / G\right)^{2}}$ in the rateindependent model is denoted $\lambda^{*}$. In Tvergaard's model, we have $\lambda^{*}=1 / 3$. We call $\lambda_{R}$ the value of $\lambda$ at complete separation, i.e. when $d=1$. We assume that $\lambda_{R}$ is dependent upon the displacement rate norm $\dot{\lambda}$. The debonding model is still written with Eqs. (3) and (4), but we may limit ourselves to a scalar expression because

$$
\|\mathbf{T}\|=E F(d)\left(v_{\mathrm{n}}^{2}+v_{\mathrm{t}}^{2}\right)^{1 / 2}=E F(d) \lambda
$$

\subsection{Homothetical approach}

We define the response for a given (controlled) constant rate $\dot{\lambda}$. The model is not modified before the peak: we have the rate-independent response. After the peak in the softening process, the first idea is to construct a homothetical response for each rate $\dot{\lambda}$. Fig. 11 shows the proposed relations by which the rate dependent softening response is obtained from the rate independent one by homothety centered on $x=\lambda^{*}$ and of ratio $\left(1-\lambda^{*}\right) /\left(\lambda_{R}-\lambda^{*}\right)$

$$
\begin{aligned}
& x=\lambda^{*}+\frac{1-\lambda^{*}}{\lambda_{R}-\lambda^{*}}\left(\lambda-\lambda^{*}\right), \\
& T=E(1-d)^{2} \lambda=E(1-x)^{2} x .
\end{aligned}
$$

By combining these two relations, we can define $d$ as a function of $\lambda$ for a constant rate $\dot{\lambda}$ (then a constant $\left.\lambda_{R}\right)$. We obtain 


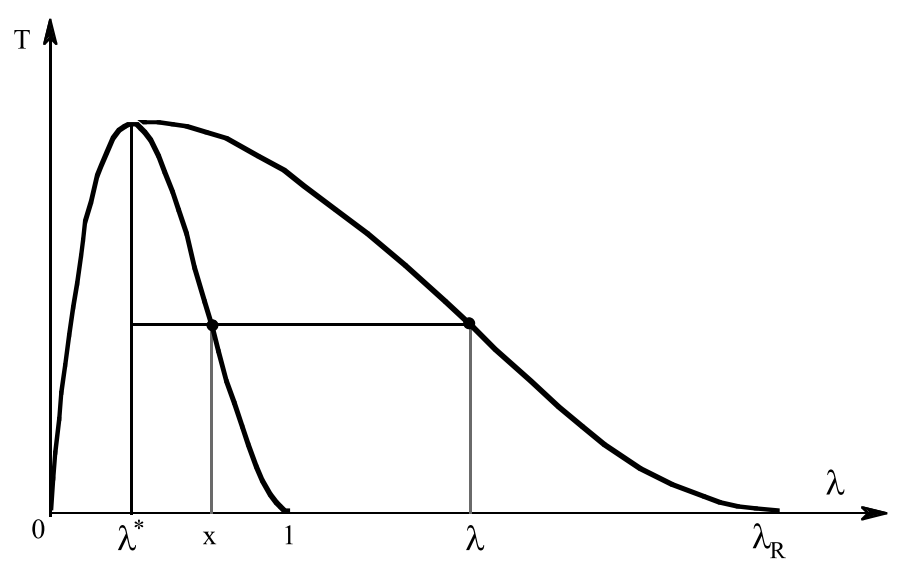

Fig. 11. Schematics of the homothetical rule for the regularization with a limited rate dependency.

$$
d=1-\frac{\left(1-\lambda^{*}\right)\left(\lambda_{R}-\lambda\right)}{\left(\lambda_{R}-\lambda^{*}\right)^{3 / 2}} \sqrt{1-\lambda^{*}+\left(\lambda_{R}-1\right) \frac{\lambda^{*}}{\lambda}} .
$$

It can be verified that for $\lambda_{R}=1$, we again have $d \equiv \lambda$ and, if $\lambda=\lambda^{*}$, we have $d=\lambda^{*}$. Moreover $\lambda=\lambda_{R}$ leads to $d=1$. We now assume the damage variation to obey the damage rate equation obtained by the direct derivation of Eq. (27) for a constant $\dot{\lambda}$. Finally, we write

$$
\begin{aligned}
& \dot{d}=\dot{\lambda} \quad \text { when } \lambda<\lambda^{*}=1 / 3 \\
& \dot{d}=\frac{1-\lambda^{*}}{\left(\lambda_{R}-\lambda^{*}\right)^{3 / 2}} \frac{2\left(1-\lambda^{*}\right) \lambda^{2}+\left(\lambda_{R}-1\right)\left(\lambda_{R}+\lambda\right) \lambda^{*}}{2 \lambda^{2} \sqrt{1-\lambda^{*}+\left(\lambda_{R}-1\right) \frac{\lambda^{*}}{\lambda}}} \dot{\lambda} \quad \text { when } \lambda>\lambda^{*} .
\end{aligned}
$$

Let us note that $\dot{d}=\dot{\lambda}$ for $\lambda=\lambda^{*}=1 / 3$, which checks the continuity of the rate.

The same damage rate equation is then used in the general case under constant or varying $\dot{\lambda}$. The function $\lambda_{R}(\dot{\lambda})$ can be defined freely, provided $\lambda_{R}(0)=1$. In the simulations, we chose the simple form

$$
\lambda_{R}(\dot{\lambda})=1+\alpha \dot{\lambda}
$$

by which the whole subspace can be defined as acceptable $\left(\lambda>x\right.$ the rate independent solution, $T \leqslant T_{\max }$ ). Saturation of $\lambda_{R}$ to a value $\lambda_{\infty}$ would lead to a more limited rate dependency, see Fig. 12, constructed with

$$
\lambda_{R}(\dot{\lambda})=\lambda_{\infty}+\left(1-\lambda_{\infty}\right) \exp \left(-\alpha \dot{\lambda} /\left(\lambda_{\infty}-1\right)\right)
$$

Fig. 12 shows the responses for constant rates $\dot{u}$ between $10^{-5}$ and $4000 \mu \mathrm{m} / \mathrm{s}$. The upper curve is the saturated one, corresponding to an infinite rate.

The choice of $\alpha$ value must be guided by the desirability to affect significantly or not the overall behavior in terms of the effect of loading rate. As shown by examples treated in Section 6, a low value for $\alpha$ allows the viscous effect to take place only at the instant of the jump, when $\dot{\lambda}$ increases to attain very high values. Then, for low values of $\alpha$, the additional energy dissipation (by viscous regularization) will be at its minimum (for a given geometry and a given discretization). 


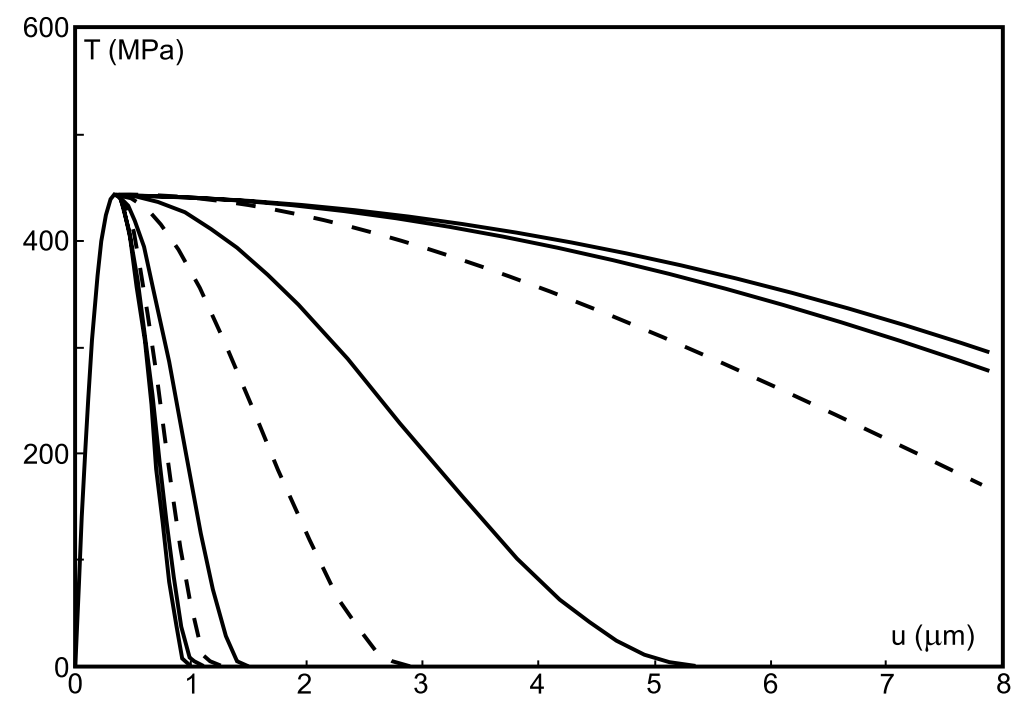

Fig. 12. Tvergaard's model regularized with the homothetical rule (Eqs. (27) and (30)). $E=3000 \mathrm{MPa}, \delta=0.2 \mu \mathrm{m}, \lambda_{\infty}=20 \mu \mathrm{m}$, $\alpha=0.1 \mathrm{~s},(-): \dot{u}=10^{-5}, 0.1,1,10,100,4000 \mu \mathrm{m} / \mathrm{s},(\cdots): \dot{u}=0.4,4,40 \mu \mathrm{m} / \mathrm{s}$.

\subsection{Logarithmic evolution}

Instead of using Eqs. (25) and (26), here we assume a simpler and direct one-to-one relation between $\lambda$ and $d$, still for a given rate $\dot{\lambda}$, that maintains the continuity and the rate continuity for $\lambda=\lambda^{*}$

$$
\begin{aligned}
& d=\lambda \text { for } \lambda<\lambda^{*}, \\
& d=\lambda^{*}+\frac{1}{\beta} \ln \left[1+\beta\left(\lambda-\lambda^{*}\right)\right] \text { for } \lambda>\lambda^{*} .
\end{aligned}
$$

Here, $\beta$ is considered to be dependent on the rate $\dot{\lambda}$. We also assume the damage rate equation by the derivation (for constant $\dot{\lambda}$ )

$$
\begin{aligned}
& \dot{d}=\dot{\lambda} \text { for } \lambda \leqslant \lambda^{*}, \\
& \dot{d}=\frac{\dot{\lambda}}{1+\beta\left(\lambda-\lambda^{*}\right)}=\mathrm{e}^{-\beta\left(d-\lambda^{*}\right)} \dot{\lambda} \text { for } \lambda>\lambda^{*} .
\end{aligned}
$$

The value of $\lambda$ at complete separation, i.e., for $d=1$, can be obtained from Eq. (31)

$$
\lambda_{R}=\lambda^{*}+\frac{1}{\beta}\left[\mathrm{e}^{\beta\left(d-\lambda^{*}\right)}-1\right] .
$$

For the Tvergaard model, if $\beta$ varies between 0 and $\beta_{\infty}=7$, the parameter $\lambda_{R}$ varies between 1 and $\lambda_{\infty} \approx 15.4$. We may assume, for instance

$$
\beta=\frac{\beta_{\infty}}{1+\beta_{\infty} / \alpha \dot{\lambda}}
$$

and the curve $T-\lambda$ only slightly overpasses the value $T_{\max }$ of the rate-independent solution, even in the large rate domain ( $T_{\max }$ is overpassed by a factor 1.25 for $\beta_{\infty}=7$ ). The present choice leads to the allowable 


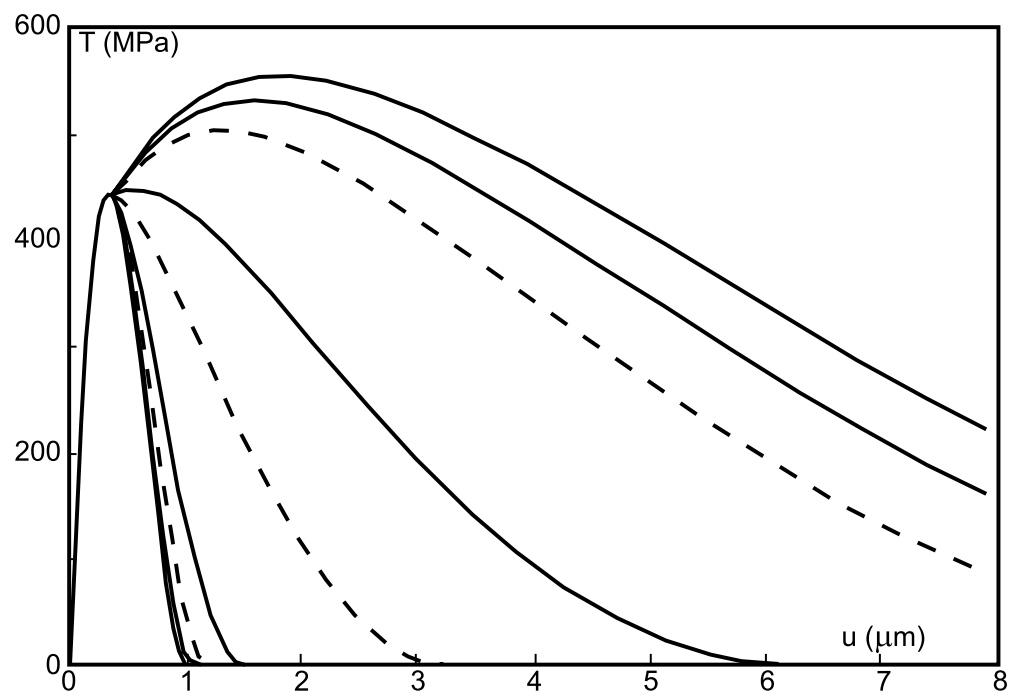

Fig. 13. Tvergaard's model regularized with the logarithmic rule (Eqs. (31) and (34)). $E=3000 \mathrm{MPa}, \delta=0.2 \mu \mathrm{m}, \beta_{\infty}=7, \alpha=2 \mathrm{~s},(-)$ : $\dot{u}=10^{-5}, 0.1,1,10,100,4000 \mu \mathrm{m} / \mathrm{s},(\cdots): \dot{u}=0.4,4,40 \mu \mathrm{m} / \mathrm{s}$.

subspace indicated in Fig. 10(b). Fig. 13 shows the local responses for the same displacement rates as in Fig. 12 (the upper curve also corresponds to the infinite rate).

\subsection{Some remarks}

(1) Let us note the thermodynamic admissibility of the modification: the independent internal state variable is $d$. Its thermodynamic affinity, not expressed here, is unchanged by the viscous regularization and is always positive (Chaboche et al., 1997b). Clearly, with the rate equations developed above, we still have $\dot{d}>0$, so that the irreversible dissipation associated with the decohesion process remains positive.

(2) The two proposed modifications are similar. The first one leads to a more complicated expression (28) for the damage rate. However, using Eq. (29), it allows the regularization for any rate, even very large. The second one is simpler but overpasses slightly the minimum viscous energy requirement and cannot eliminate jump problems for very high rates, at least in the elementary uniaxial example. In practice, when used in numerical simulations, with spatial redistributions, they deliver similar results, the additional energy dissipated by viscosity being essentially the minimum required in order to smooth the jump.

\section{Applications and convergence tests}

In order to check the capabilities of the regularization method, we designed several numerical tests of different natures. First, the uniaxial problem with only one element that stores the energy before the rapid decohesion, second, a DCB example, and third, the initiation and growth of decohesion at the fiber/matrix interface in a unidirectional composite submitted to a transverse tensile loading. In all regularized solutions we used the "homothetical form" of Section 6.3, with relation (29). 


\subsection{Uniaxial "jump problem” simulations}

We consider the simplest structure studied in Section 3.1 (Fig. 3a), where the upper elastic element stores elastic energy before its sudden release when the debounding takes place at the interface element. We compare the regularization properties of the LMT model and the model proposed in Section 5.1 (the homothetical rule), using the linear dependency (29) between the displacement rate $\dot{\lambda}$ and the displacement at complete separation $\lambda_{R}$. The numerical responses of the two models are adjusted to each other at the lowest rate considered, $\dot{U}=0.01 \mathrm{~mm} \mathrm{~s}^{-1}$, with a compromise between equal maximum tensions, similar dissipated energies, and similar displacements at the time of complete separation. Fig. 14 shows this adjustment for the lowest rate. The model parameters are $E=1800 \mathrm{MPa}, \delta=0.1 \mu \mathrm{m}$ and $\alpha=11.5 \mathrm{~s}$ for the Tvergaard regularized model (with Eqs. (28) and (29)), and $E=600 \mathrm{MPa}, \delta=0.1 \mu \mathrm{m}$ and $a=1$ for the LMT model (the value $\alpha=11.5 \mathrm{~s}$ is due to the adjustment of the dissipated energy to the one dissipated by LMT model at the lowest rate). Let us note that for the rate-independent solutions, the two models will have a "solution jump" as long as the stiffness set for the adjacent elastic element is $k=E_{0} / L=0.1$ (N $\mathrm{m}^{-1}$ ). With the two models, the solution jump is suppressed for every control rate $\dot{U}$. For the lowest ones, we observe that the additional (viscous) energy dissipated is exactly the energy difference between elastic energy stored in the adjacent part before the solution jump (in the rate independent case) and the energy dissipated by decohesion. In fact, when the jump situation occurs, the rate $\dot{\lambda}$ increases dramatically, which increases the value of $\lambda_{R}(\dot{\lambda})$ as well, then the corresponding strength, in such a manner to always respect the solution continuity.

For larger control rates $\dot{U}$, Fig. 15 illustrates the different responses in the two models, in terms of the total energy dissipated before failure. For high rate conditions, the rate dependency appears as linear in our model but quadratic with the Allix-Ladevèze model, which can be easily understood from the local properties of the interface models.

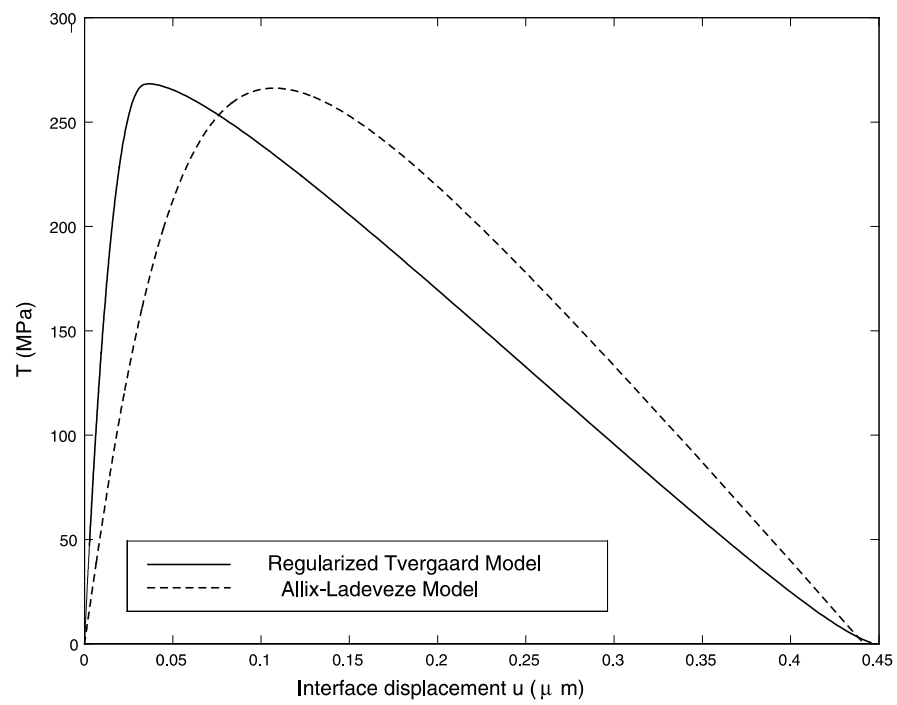

Fig. 14. Application of two regularized models to the simple "two elements" structure of Fig. 2: (-) proposed regularization of the Tvergaard model. (- - -) Allix-Ladevèze model. 


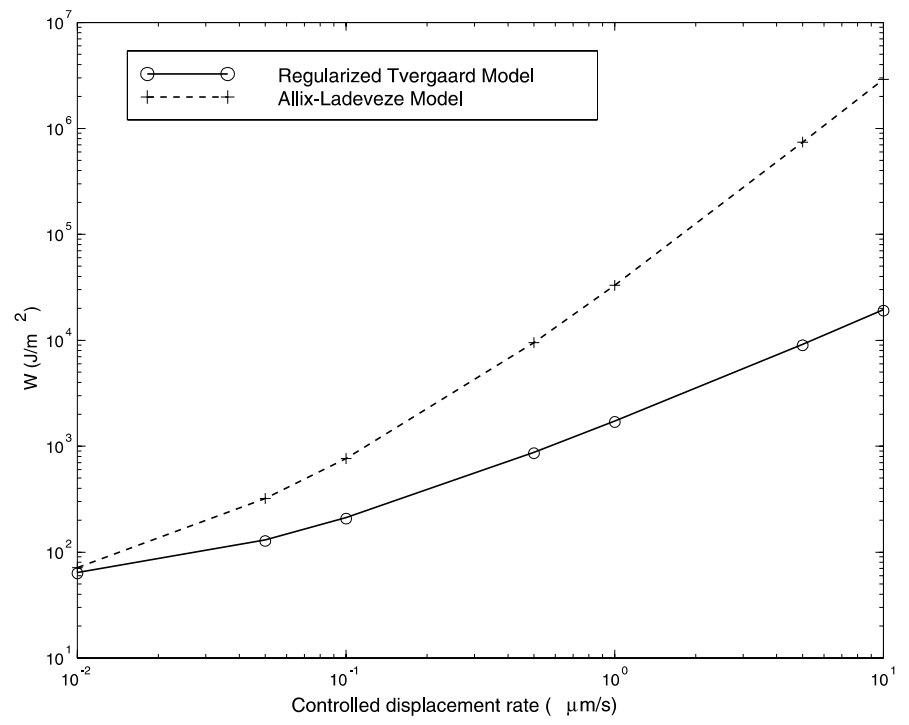

Fig. 15. Energy dissipated before complete separation as a function of the controlled displacement rate $\dot{U}:(-$ o -$)$ proposed regularization of the Tvergaard model. (- + -) Allix-Ladevèze model.

\subsection{Example of a double cantilever beam}

The second example, more similar to a "real" structural application, is the delamination growth in a DCB component. Its length is 20 and its total thickness $4 \mathrm{~mm}$. Two regular meshes have been selected, one with quadratic rectangular elements, $0.250 \times 0.200 \mathrm{~mm}^{2}$, shown in Fig. 16, the second with $0.125 \times 0.100$ $\mathrm{mm}^{2}$. The upper and lower parts are considered to be elastic isotropic $\left(E_{0}=400 \mathrm{GPa}, v=0.19\right)$ and the interface is defined by $E=G=2000 \mathrm{MPa}, \delta_{\mathrm{n}}=\delta_{\mathrm{t}}=0.001$ for the Tvergaard model. The loading is controlled with the displacement of the corner nodes, at a constant rate $\dot{U}=0.003 \mathrm{~mm} \mathrm{~s}^{-1}$. The solutions with this rate independent model are given by the broken lines in Fig. 17. It is worth noting that a sudden load drop takes place at the beginning of the non-linear behavior, when damage becomes significant at the first interface node, corresponding to the crack initiation. Other subsequent jumps can be observed, more or less regularly spaced, corresponding to the discrete mesh. The finer mesh clearly shows the first jump at initiation, followed by a practically continuous response. Let us note the difference in the initial elastic stiffness of the load-displacement response, due to the mesh dependency.

On the other hand, the viscous regularization proposed in Section 5.1 comes out in Fig. 17 as continuous lines, using $\alpha=0.5 \mathrm{~s}$ in Eq. (29), chosen more or less arbitrarily. This means that the load varies contin-

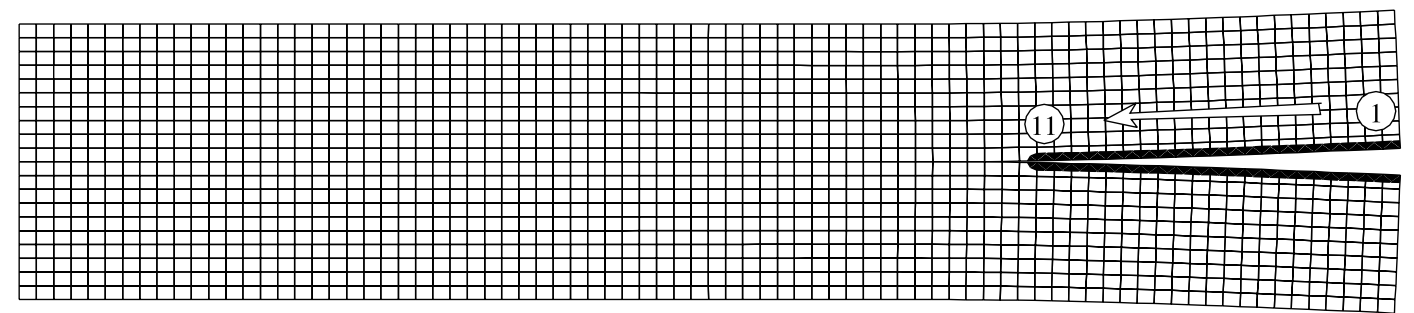

Fig. 16. The mesh for the Double Cantilever Beam $\left(0.250 \times 0.200 \mathrm{~mm}^{2}\right)$. Numbers indicate interface node positions for Fig. 19 . 

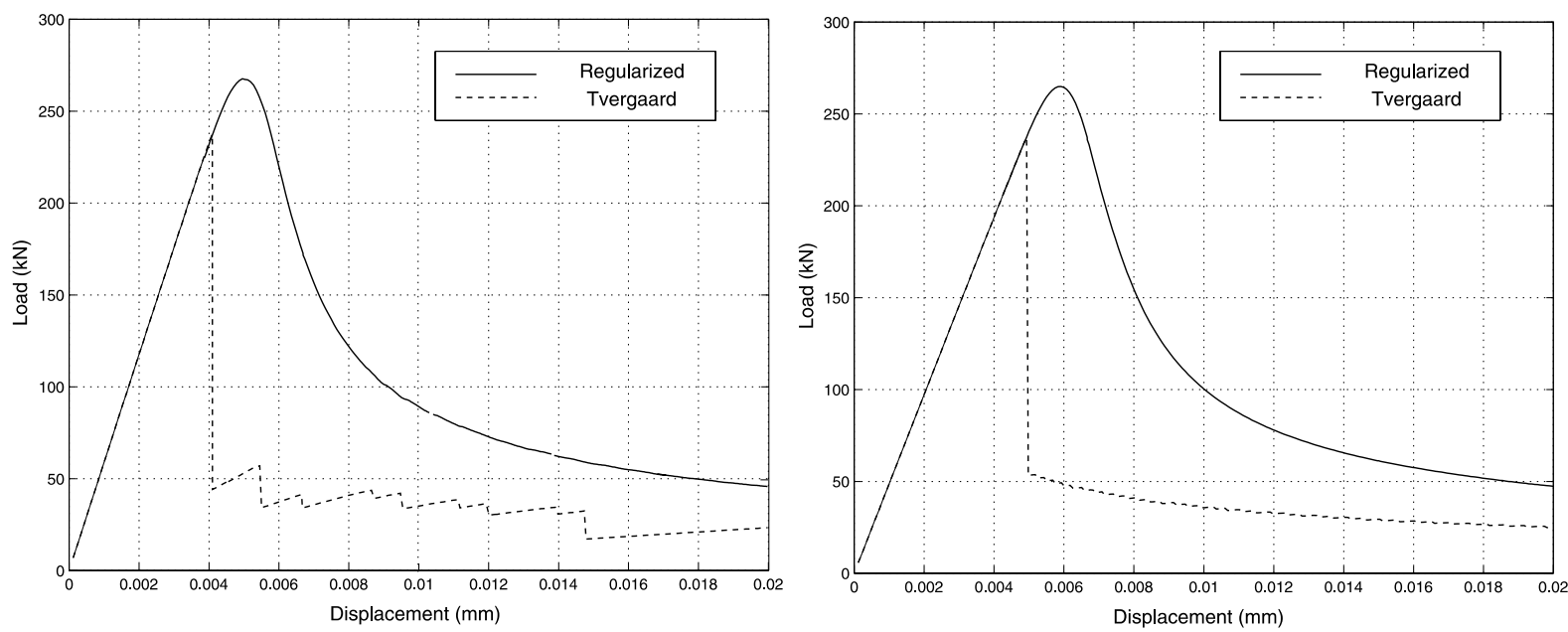

Fig. 17. Comparison of the force-displacement responses, with Tvergaard's rate independent model and the regularized one, on the DCB with a controlled displacement $\dot{U}=0.003 \mathrm{~mm} \mathrm{~s}^{-1}$. Left: $0.250 \times 0.200 \mathrm{~mm}^{2}$ mesh size, right: $0.125 \times 0.100 \mathrm{~mm}^{2} \mathrm{mesh}$ size .

uously, with no jump. As the same set of parameters is used for the rate-independent part in Fig. 17, we obviously observe a greater dissipated energy in the viscous case. A better comparison could have been obtained by readjusting the parameters $E$ and $\delta$ in the second case. Fig. 18 shows the comparison of the two regularized solutions, eliminating the initial elastic stiffness difference between the two meshes (a multiplication of the coarse mesh displacement solution by a constant factor). Further study is necessary in order to quantify the effect of the viscous regularization more precisely in terms of material parameters, structural applications, and loading rate effects.

Moreover, Fig. 19 gives the evolution of the local normal traction at the interface for points taken for every two elements in the mesh of Fig. 16. Point 1 corresponds to the first interface node. We clearly

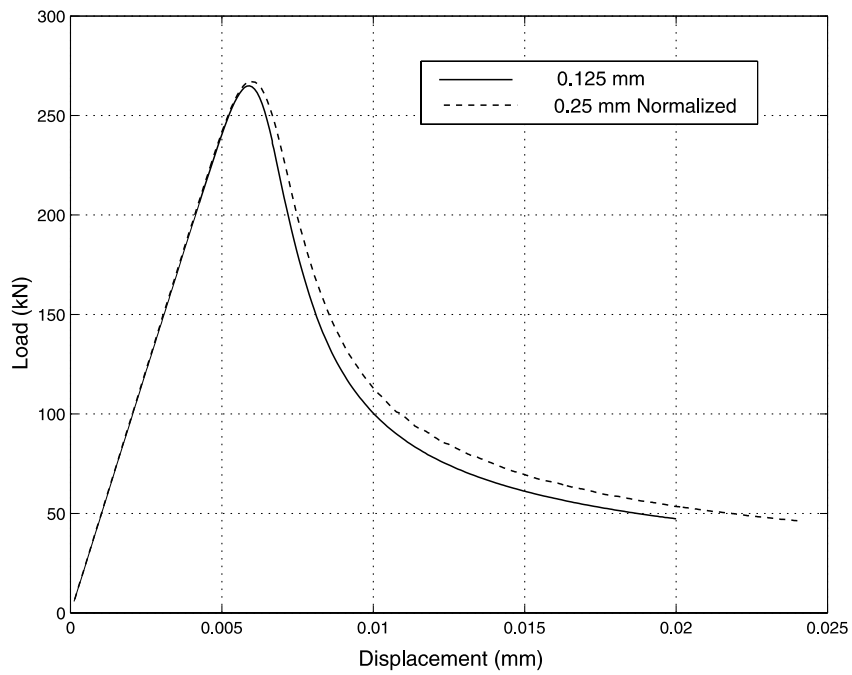

Fig. 18. DCB responses with the two regularized solutions, when normalizing the coarse mesh initial elastic stiffness by the fine mesh stiffness. 


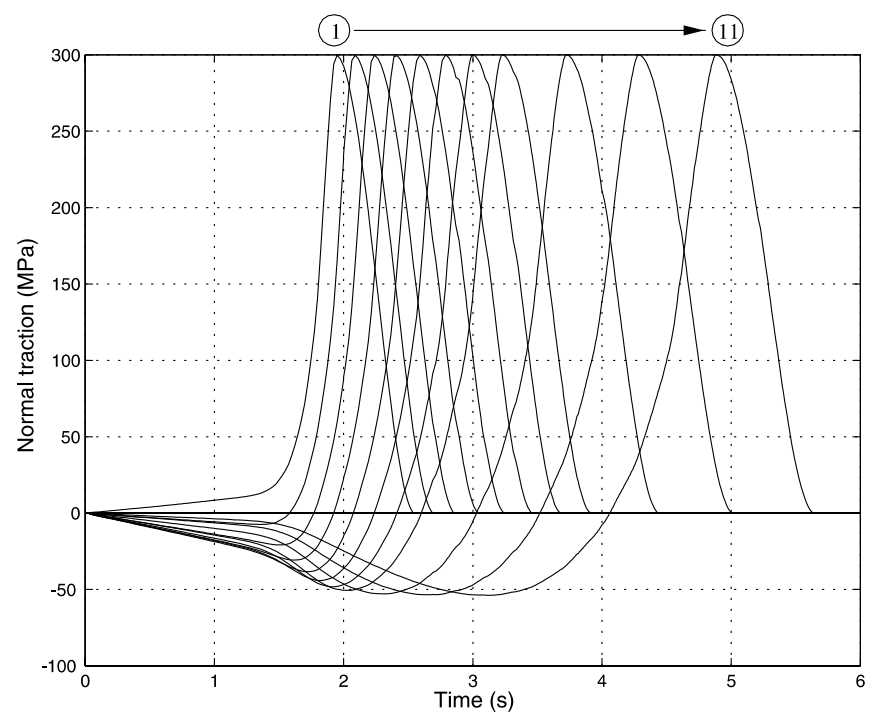

Fig. 19. Local evolutions of the normal interface traction on eleven interface nodes of the DCB (coarse mesh).

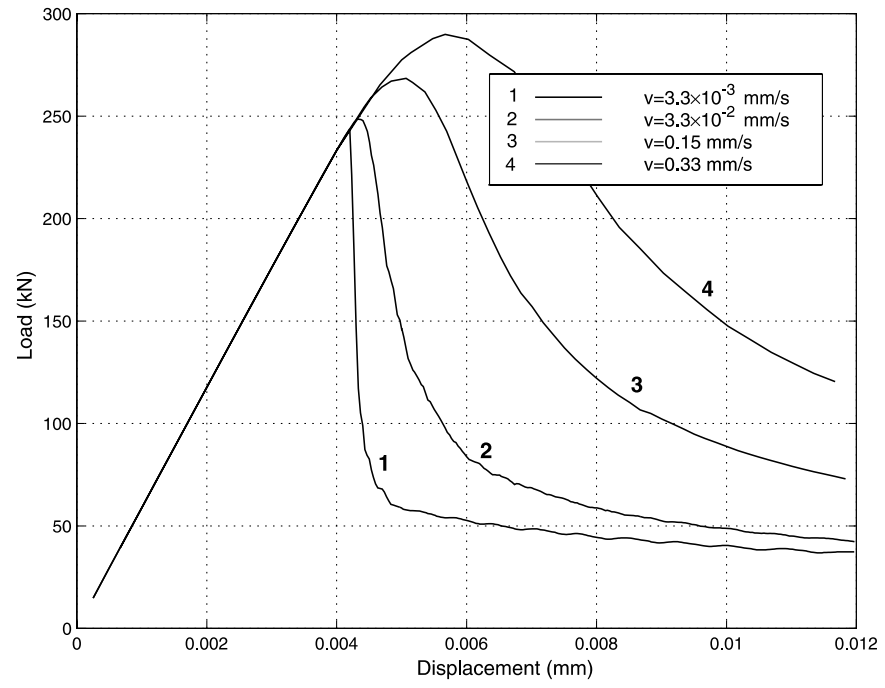

Fig. 20. DCB responses with the regularized model for various controlled displacement rates (coarse mesh).

observe a local compression state ahead of the "equivalent crack tip" after the first decohesion, then traction takes place, followed by progressive decohesion, softening and complete separation (zero traction), with perfectly continuous evolutions, thanks to the regularization.

Fig. 20 shows the DCB response for various controlled displacement rates, applied on the coarse mesh. The parameter $\alpha$ for the regularized model (Eq. (29)) is taken as $0.01 \mathrm{~s}$. In every case, responses are continuous, but with the lower rate $\left(0.33 \times 10^{-2} \mathrm{~mm} \mathrm{~s}^{-1}\right)$, the load drops very rapidly, like in the rate independent simulation. As the rate increases, the load increases slightly (only $15 \%$ for a rate factor of 100) and the total dissipated energy varies by a factor of about 2.5 . 

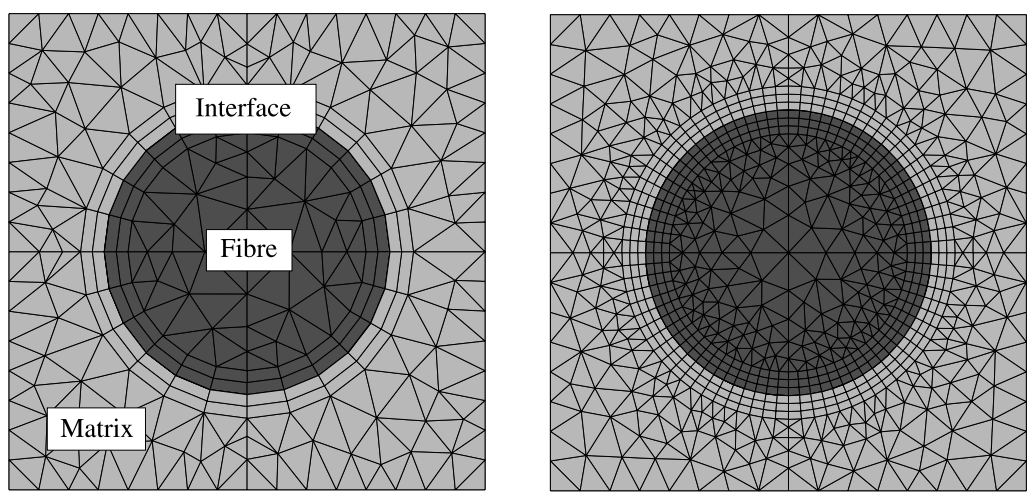

Fig. 21. Coarse and fine meshes of the unit cell for a 30\% fiber volume fraction on a UD composite system.

\subsection{Debounding at the fiber/matrix interface}

A test example has been designed that corresponds to the debounding at the fiber/matrix interface in a unidirectional composite system. A square unit cell is treated, in the framework of periodic homogenization method (Feyel, 1998). The two meshes used are given in Fig. 21 for a 30\% fiber volume fraction. The coarse mesh has 299 nodes, 412 quadratic elements and 24 interface elements. The corresponding numbers for the fine mesh are respectively 1044, 1460, 80. Fiber and matrix are elastic isotropic with the following values: $E_{\mathrm{f}}=75 \mathrm{GPa}, v_{\mathrm{f}}=0.3, E_{\mathrm{m}}=400 \mathrm{GPa}, v_{\mathrm{m}}=0.19$. The interface elements are treated with Tvergaard's model, using $E=G=2025 \mathrm{MPa}, \delta_{\mathrm{n}}=\delta_{\mathrm{t}}=1 \mu \mathrm{m}$. The regularization parameter $\alpha$ of Eq. (29) is taken as $0.01 \mathrm{~s}$, that corresponds to a very low value: it will affect the overall rate dependency only for very high loading rates.

The unit cell is submitted to a pure uniaxial tensile loading, under a strain control. In the periodic homogenization technique, we control the overall strain $E_{11}$, and compute the average stress $\Sigma_{11}$ over the unit cell. The overall stress-strain responses are given on Figs. 22-24. As expected there is a solution jump for the rate independent model as seen on Fig. 22 (in only one time step). This discontinuity takes place even for the very fine mesh. This result is very different than the one observed for the pure bending example of Section 3.3. In the present case, reducing the mesh size does not eliminate the solution jump.

In Fig. 23, we compare the rate independent simulation and the regularized one, for the coarse mesh: the two responses are very similar, except the continuous solution in the second (intermediate time steps, clearly shown with crosses) when the sudden debonding occurs. Fig. 24 compares the responses obtained with the regularized model for three overall strain rates 1,10 and $100 \mathrm{~s}^{-1}$. The rate effect is really very limited for the overall response, thanks to the low $\alpha$ value. After the first sudden stress drop, secondary drops take place when the debonded zone progresses along the fiber/matrix interface.

\section{Conclusion}

Some interface debonding models have been briefly recalled, that have the capability of describing the progressive loss of adhesion. Special attention has been focused on the numerical instability problems induced by the possibility of solution jumps in the rate independent models. The main results are as follows:

- The "solution jump" can arise for every rate independent debonding model, depending on the material parameters in regards of the analyzed structure. 


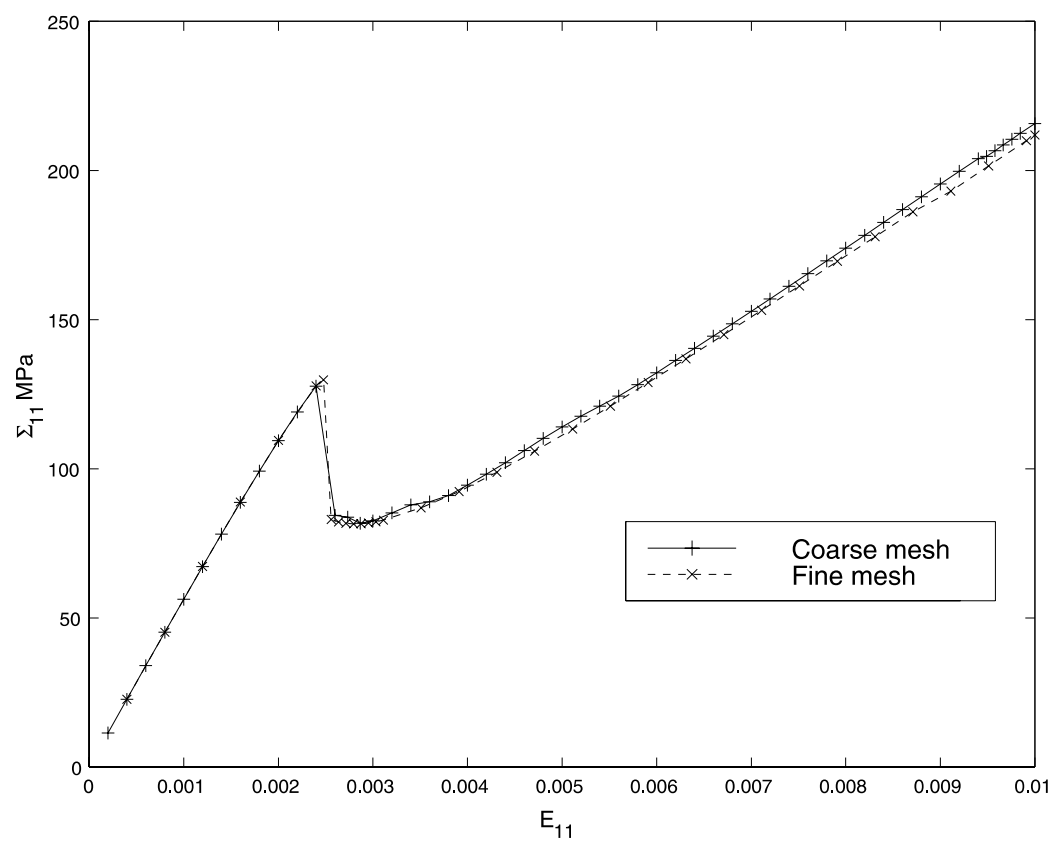

Fig. 22. Overall uniaxial stress-strain response of the UD composite system for the two meshes and the rate independent interface debonding model (fiber and matrix are purely elastic).

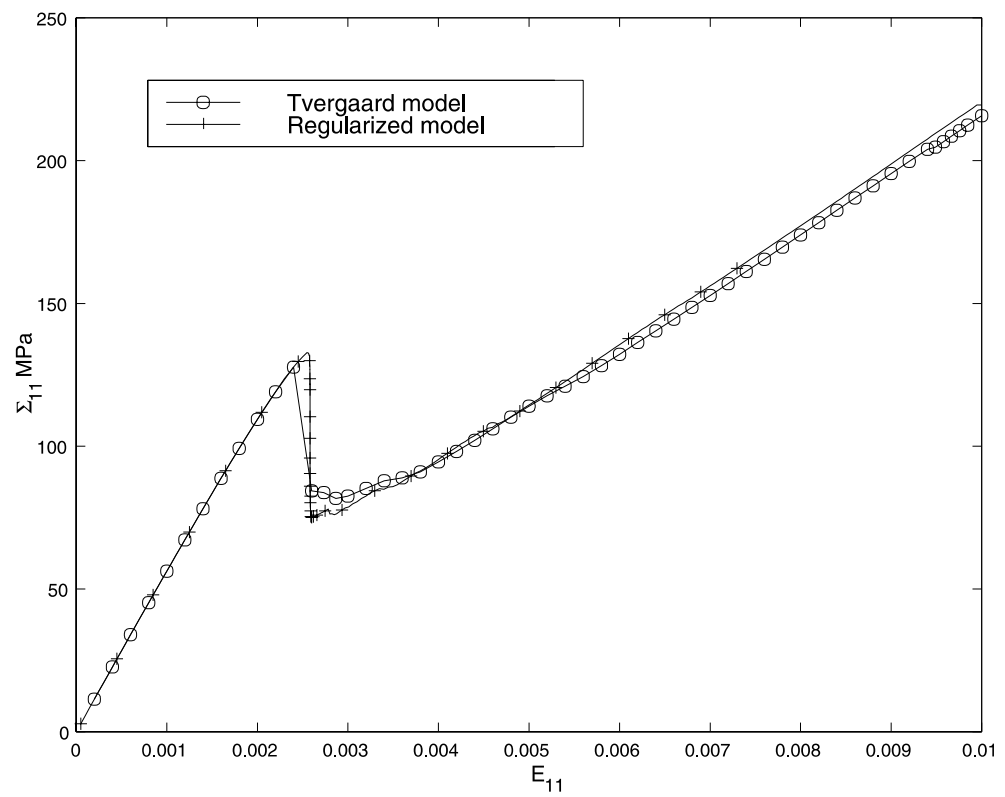

Fig. 23. Comparison of the rate independent and regularized solutions for the transverse traction on the UD composite system (coarse mesh). 

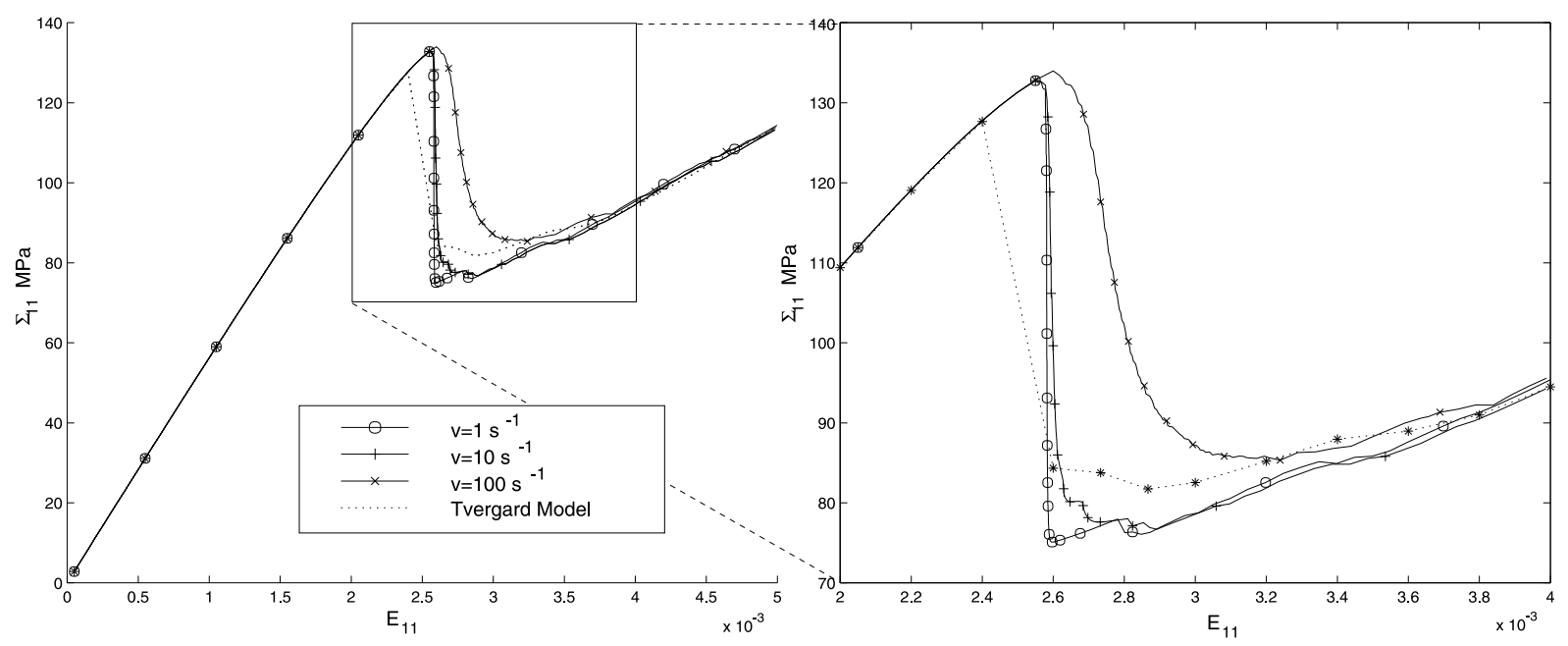

Fig. 24. Overall responses for the transverse traction on the UD composite system with the rate independent version and several overall rates with the regularized version.

- It is related to the elastic energy stored in the surrounding of the damaging interface element. It is a mesh dependent effect.

- Some interface models are written in a viscous format that eliminates the possibility of solution jumps but induces a great loading rate dependency in the structural analysis.

- A better compromise can be found using a viscous regularization with a limited rate dependency. This is sufficient to eliminate any solution jump but with a much lower rate effect.

The models have been implemented in a finite element code, both in their rate independent and viscous versions. The example of a DCB delamination analysis has demonstrated the capability of the new modified model. Further work is needed in order to assess the rate dependency in various structural examples, in comparison with experimental data, especially on ceramic matrix composites. The model will be applied to true situations involving push-in, push-out, pull-out micro-mechanical tests and transverse loadings on unidirectional composites.

At this level, a question is still open: what is the status of the energy released? In the case of an instantaneous "solution jump", as in the simple example of Section 3.1, a "fictitious energy" part under the tension-displacement curve (in the local framework) was not really dissipated. On the other hand, with the viscous regularization proposed, even if it is limited to a minimum, an additional viscous energy is dissipated, and this must be taken into account when identifying with the fracture energy obtained from experiments. Some additional results are given by Monerie et al. (1998) concerning the rate dependency of these different viscous models into a structural application where fiber/matrix interface loading combines shear and normal pressure.

The present paper considered numerical aspects only. In terms of experimental trends, several situations could be expected

- For delamination in laminate composite structures, for instance in tests like the DCB, the initiation stage often takes place suddenly with a significant and quasi-instantaneous load drop. In such cases the proposed regularization technique would introduce a non-realistic additional dissipated energy. For that reason, other techniques are under study among those mentioned in Section 3.4. 
- Contrarily, the delamination growth is most often a continuous process and the proposed method could be useful in that context, in order to overcome the unrealistic and mesh dependent secondary load jumps.

- In the context of fiber-matrix interfacial debonding in composites, the two situations can be observed experimentally at the onset of debonding: either a sudden load drop or a continuous response (with or without a load decrease), depending on the elastic energy stored in the surrounding before initiation. In such cases, the regularization method should also be considered as a good tool.

\section{Appendix A. Modified Tvergaard model}

The Tvergaard model has been modified by Chaboche et al. (1997a,b) in order to smoothen the strength transition, under normal compression/tangential loading, when the complete separation takes place and Coulomb's friction begins to play role. This modified model is briefly recalled, for the general 3D version, in its rate independent format. It can be written in a thermodynamic framework, which is not considered here.

We use the displacement differences across the interface denoted $u_{\mathrm{n}}$, $\mathbf{u}$, where $\mathbf{u}$ is the tangential displacement vector of components $\left(u_{\mathrm{t}}, u_{\mathrm{b}}\right)$, and the corresponding normalized displacements $v_{\mathrm{n}}=u_{\mathrm{n}} / \delta_{\mathrm{n}}$, $\mathbf{v}$ of components $v_{\mathrm{t}}=u_{\mathrm{t}} / \delta_{\mathrm{t}}, v_{\mathrm{b}}=u_{\mathrm{b}} / \delta_{\mathrm{b}}$. For the sake of simplicity, we assume identical material characteristics in the two tangential directions, which means $\delta_{\mathrm{b}}=\delta_{\mathrm{t}}$ and $G_{\mathrm{b}}=G_{\mathrm{t}}=G$.

The model introduces a tangential inelastic slip, obeying Coulomb's friction, as soon as gradual damage begins. This effect is modeled by way of a tangential "plastic" displacement parameter $\mathbf{v}^{\mathrm{p}}=\mathbf{u}^{\mathrm{p}} / \delta_{\mathrm{t}}$. With these notations, the normal and tangential tensile forces at the interface express

$$
\begin{aligned}
& T_{\mathrm{n}}=\left[E F(d) H\left(v_{\mathrm{n}}\right)+K H\left(-v_{\mathrm{n}}\right)\right] v_{\mathrm{n}}, \\
& \mathbf{T}=G\left(\mathbf{v}-\mathbf{v}^{\mathrm{p}}\right),
\end{aligned}
$$

where $E$ and $G$ are the initial normal and tangential stiffnesses, and $K$ is the compressive stiffness (with an arbitrarily high value that limits penetration). $H$ is the Heaviside function and $F(d)$ is the damage function that affects the stiffness. The two irreversible processes are described, through the Coulomb criterion

$$
f=\left\|\mathbf{T}-\frac{G F(d)}{1-F(d)} \mathbf{v}^{\mathrm{p}}\right\|-\left\langle-\mu T_{\mathrm{n}}\right\rangle \leqslant 0
$$

and a rate independent damage rule

$$
g=\lambda\left(v_{\mathrm{n}}, \mathbf{v}\right)-d \leqslant 0,
$$

where $\lambda$ represents a given scalar function of the present displacements and $d$ is the scalar damage variable, in fact the maximum value attained by $\lambda$. The key equation in the model corresponds to the choice of the function $\lambda$ (arguments are given in Chaboche et al. (1997b))

$$
\lambda=\left[\left\langle v_{\mathrm{n}}\right\rangle^{2}+\left\|\mathbf{v}-\frac{\mu K}{G}\left\langle-v_{\mathrm{n}}\right\rangle \mathbf{n}\right\|^{2}\right]^{1 / 2},
$$

where $\mathbf{n}$ is the present unit direction of sliding. $\mathbf{n}$ expresses by combining Eqs. (A.2) and (A.3)

$$
\mathbf{n}=\left[\mathbf{v}-\frac{\mathbf{v}^{\mathrm{p}}}{1-F(d)}\right] /\left\|\mathbf{v}-\frac{\mathbf{v}^{\mathrm{p}}}{1-F(d)}\right\|
$$

There are several situations, depending on the instantaneous loading conditions (assumed here under displacement control). They are illustrated by Fig. 25: 


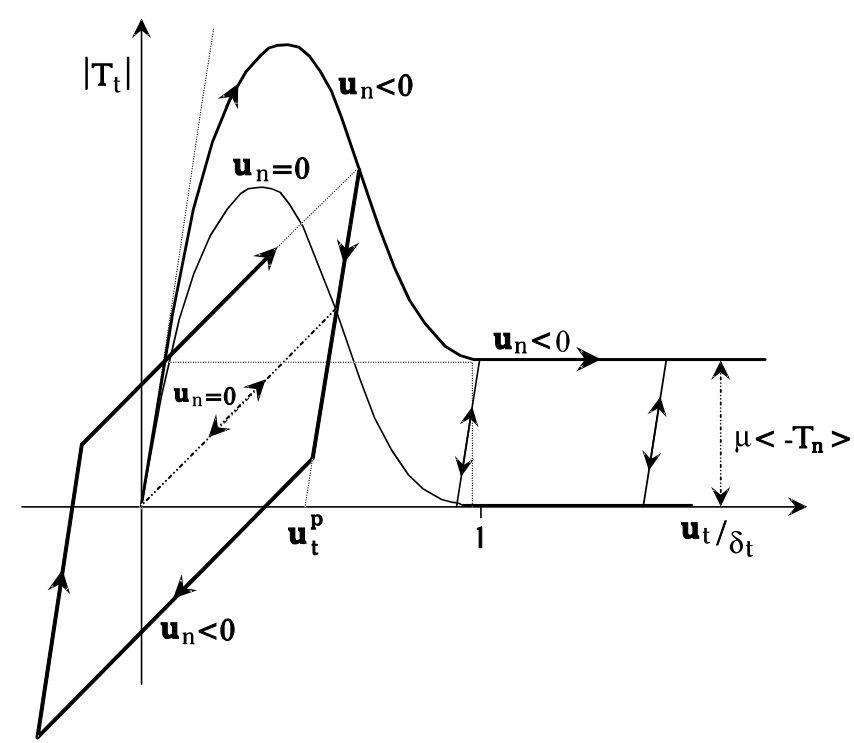

Fig. 25. Schematic behavior of the ONERA version of Tvergaard's model under tangential loading.

- With a normal tension $\left(v_{\mathrm{n}}>0\right)$, the fact that $T_{\mathrm{n}}>0$ from Eq. (A.1) and the need for Eq. (A.3) induces automatically

$$
\mathbf{T}=G F(d) \mathbf{v}
$$

and the model reduces exactly to Tvergaard's one (Fig. 1).

- With a normal compression and "elastic unloading" ( $v_{\mathrm{n}} \leqslant 0$ and $f<0$ and $g<0$ ), we have constant $\mathbf{v}^{\mathrm{p}}$ and $d$ and the response is given by Eqs. (A.1) and (A.2). Let us note the tangential elastic response that uses the initial stiffness $G$.

- With a normal compression, "slip condition" but "no damage growth" ( $v_{\mathrm{n}} \leqslant 0$ and $f=0$ and $g<0$ ), we use Eqs. (A.1)-(A.3). The evolution of $\mathbf{v}^{\mathrm{p}}$ is given via Coulomb's friction rule $\left(v_{\mathrm{n}}^{\mathrm{p}}=0\right)$

$$
\dot{\mathbf{v}}^{\mathrm{p}}=\dot{\xi} \frac{\mathbf{T}-\mathbf{X}}{\|\mathbf{T}-\mathbf{X}\|}, \quad \mathbf{X}=\frac{G F(d)}{1-F(d)} \mathbf{v}^{\mathrm{p}},
$$

where $\xi$ is given by the consistency condition $\dot{f}=0$. Fig. 25 illustrates the corresponding "linear kinematic hardening", whose slope is depending on the previously developed damage.

- With a normal compression and simultaneous "slip and damage growth", we use Eqs. (A.4) and (A.5) to define the damage evolution. Let us note that damage without slip is not allowed in compression with the present model.

\section{Appendix B. Other interface debounding models}

\section{B.1. The model of Allix and Ladevèze}

The version presented here is taken from the recent paper by Allix and Ladevèze (1996). It is written in a standard thermodynamic framework using a state potential that involves the stresses. Moreover, this 
potential is assumed to depend on three damage variables $d_{\mathrm{n}}, d_{\mathrm{t}}, d_{\mathrm{b}}$ associated with the tensile and tangential directions, respectively. Using the same notation as above, we obtain the displacements-tensile relations

$$
u_{\mathrm{n}}=\frac{1}{K_{\mathrm{n}}}\left[\frac{\left\langle T_{\mathrm{n}}\right\rangle}{1-d_{\mathrm{n}}}+\left\langle-T_{\mathrm{n}}\right\rangle\right], \quad u_{\mathrm{t}}=\frac{T_{\mathrm{t}}}{K_{\mathrm{t}}\left(1-d_{\mathrm{t}}\right)}, \quad u_{\mathrm{b}}=\frac{T_{\mathrm{b}}}{K_{\mathrm{b}}\left(1-d_{\mathrm{b}}\right)},
$$

where $K_{\mathrm{n}}, K_{\mathrm{t}}, K_{\mathrm{b}}$ are the corresponding interface stiffnesses. The damage evolution is constructed from the thermodynamic forces associated to the damage components, respectively

$$
\begin{aligned}
& y_{\mathrm{n}}=\frac{1}{2} \frac{\left\langle T_{\mathrm{n}}\right\rangle^{2}}{K_{\mathrm{n}}\left(1-d_{\mathrm{n}}\right)^{2}}=\frac{1}{2} K_{\mathrm{n}}\left\langle v_{\mathrm{n}}\right\rangle^{2}, \\
& y_{\mathrm{t}}=\frac{1}{2} \frac{T_{\mathrm{t}}^{2}}{K_{\mathrm{t}}\left(1-d_{\mathrm{t}}\right)^{2}}=\frac{1}{2} K_{\mathrm{t}} u_{\mathrm{t}}^{2}, \\
& y_{\mathrm{b}}=\frac{1}{2} \frac{T_{\mathrm{b}}^{2}}{K_{\mathrm{b}}\left(1-d_{\mathrm{b}}\right)^{2}}=\frac{1}{2} K_{\mathrm{b}} u_{\mathrm{b}}^{2}
\end{aligned}
$$

and from an equivalent damage energy release rate

$$
Y=\sup _{\tau \leqslant t}\left[y_{\mathrm{n}}^{\alpha}+\left(\gamma_{\mathrm{t}} y_{\mathrm{t}}\right)^{\alpha}+\left(\gamma_{\mathrm{b}} y_{\mathrm{b}}\right)^{\alpha}\right]^{1 / \alpha}
$$

Then, the damage evolution is restricted to have $d_{\mathrm{n}}=d_{\mathrm{t}}=d_{\mathrm{b}}=d$ during the whole history, obeying the criterion (Ladevèze, 1992)

$$
d=\omega(Y)=\frac{\left\langle\sqrt{Y}-\sqrt{Y_{0}}\right\rangle}{\sqrt{Y_{c}}-\sqrt{Y_{0}}} .
$$

In Eqs. (B.2) and (B.3), $\gamma_{\mathrm{t}}, \gamma_{\mathrm{b}}, \alpha$ and $Y_{0}, Y_{c}$ are material parameters. In particular, $\gamma_{\mathrm{t}}$, and $\gamma_{\mathrm{b}}$ make it possible to satisfy mode II and mode III decohesion independently, and $\alpha$ describes the shape of the fracture mechanics failure loci. A somewhat more complex expression for $\omega(Y)$, not retained here, was used in Allix and Ladevèze (1996). Moreover, possibilities for inelastic effects due to friction are also present in the model.

Let us point out a few differences with the modified Tvergaard model presented in Appendix A:

- from Eq. (B.1a), we observe the same stiffness $K_{\mathrm{n}}$ for normal tension and normal compression, which may induce larger penetrations,

- the damage criterion is slightly different, due to the separation of the three different modes in Eq. (B.3) and to the additional degree of freedom given by exponent $\alpha$,

- a damage threshold, given by $Y_{0}$ has been implemented,

- the effect of damage on the elastic behavior is given by a linear function $(1-d)$ instead of the quadratic function $F(d)=(1-d)^{2}$ in Tvergaard's model.

The last difference is for the use of a delay effect in the damage evolution rule. It consists in replacing Eq. (B.4) by the following:

$$
\dot{d}=\frac{\eta}{a}[1-\exp (-a\langle\omega(Y)-d\rangle)] .
$$

The inviscid solution, for an infinitely low rate, reconstructs (B.4). The delay effect is defined by the characteristic time $a / \eta$. With this model, a maximum damage rate is given by $\eta / a$. 
This model combines strict inequalities that describe the unilateral contact/Coulomb's friction condition with an equation for the decohesion process. It was formulated within a quasi-standard thermodynamic framework (Cangémi, 1997) that will not be discussed here. We use the same notation as in Appendix A. Instead of Eq. (A.1) above, we have, for normal behaviour, through Signorini's equations

$$
T_{\mathrm{n}}-c_{\mathrm{n}} F(d) u_{\mathrm{n}} \leqslant 0, \quad u_{\mathrm{n}} \geqslant 0, \quad\left[T_{\mathrm{n}}-c_{\mathrm{n}} F(d) u_{\mathrm{n}}\right] u_{\mathrm{n}}=0
$$

The first difference with the modified Tvergaard model (Appendix A) is that the non-penetration condition is satisfied strictly. For the tangential behavior, we have Coulomb's condition in the following form:

$$
f=\left\|\mathbf{T}-c_{\mathrm{t}} F(d) \mathbf{u}\right\|-\mu\left|T_{\mathrm{n}}-c_{\mathrm{n}} F(d) u_{\mathrm{n}}\right| \leqslant 0,
$$

where the interface stiffnesses $c_{\mathrm{n}}$ and $c_{\mathrm{t}}$ could be equal in practice. The second difference is the purely inelastic tangential behavior $\left(\mathbf{u} \equiv \mathbf{u}^{\mathrm{p}}\right.$ ), so that the model works as a rigid-plastic model for the tangential components (with a similar kinematic hardening as before).

The third difference is given by the decohesion growth equation itself, with $F(d)=(1-d)^{2}$ :

$$
\dot{d}=\frac{1}{b}\left\langle(1-d)\left(c_{\mathrm{n}} u_{\mathrm{n}}^{2}+c_{\mathrm{t}}\|u\|^{2}\right)-w\right\rangle
$$

In that equation, there appears a threshold $w$, under which no damage takes place. Moreover, Eq. (B.8) has a clear viscous character ( $b$ coefficient of viscosity), which leads to the rate dependency in Section 4.

\section{Appendix C. Elementary solutions and comparisons}

For comparison purposes, we explicit the models in their simplest uniaxial form (scalar) and give, when possible, closed form solutions for the monotonic loading case. In other cases, the solutions are obtained numerically.

\section{C.1. Rate-independent limit case}

The Tvergaard model immediately becomes

$$
T=E F\left(\frac{u}{\delta}\right) \frac{u}{\delta}=E\left(1-\frac{u}{\delta}\right)^{2} \frac{u}{\delta} .
$$

$T$ and $u$ being, respectively, the positive traction and the corresponding displacement across the interface, while $E$ and $\delta$ are the two parameters. In the model by Allix and Ladevèze, we have $y=(1 / 2) K u^{2}$ and, from Eq. (B.4) we find

$$
d=\omega(Y)=\frac{\left\langle u-u_{0}\right\rangle}{u_{c}-u_{0}}
$$

where $u_{0}$ and $u_{c}$ are, respectively, the displacement at the onset of damage growth and the value at complete separation. The traction displacement relation then expresses

$$
T=K(1-d) u=K\left[1-\frac{\left\langle u-u_{0}\right\rangle}{u_{c}-u_{0}}\right] u .
$$

For the model by Cangémi et al. (1995), we need to establish the inviscid solution for an infinitely slow increasing displacement $(\dot{u} \rightarrow 0)$. In that case, Eq. (B.8) gives $c(1-d) u^{2}=w$ and with the notation $\sigma_{0}=c u_{0}=\sqrt{w c}$, we find (from the equality in Eq. (B.6a) and for $u \geqslant u_{0}$ ) 


$$
T=c(1-d)^{2} u=\frac{w^{2}}{c} u^{-3}=\sigma_{0}\left(\frac{u}{u_{0}}\right)^{-3} .
$$

Then, the traction-displacement relation expresses

$$
\begin{aligned}
& T=c u \quad \text { if } u \leqslant u_{0}, \\
& T=\sigma_{0}\left(\frac{u}{u_{0}}\right)^{-3} \quad \text { if } u \geqslant u_{0} .
\end{aligned}
$$

We note in that case, the infinite displacement for complete separation $(T \rightarrow 0)$.

The three models can be adjusted to each other approximately. In Fig. 26, we have considered the same maximum tension and the same fracture energy (fracture energy can be calculated analytically by the integrals under the tension-displacement curves). We find, respectively $\left(\xi=u_{0} / u_{c}\right.$ in the Allix-Ladevèze model),

$$
\begin{aligned}
& T_{\max }=\frac{4}{27} E=\frac{1}{4} \frac{K u_{c}}{1-\xi}=\sigma_{0}=\sqrt{w c}, \\
& G_{c}=\frac{1}{12} E \delta=\frac{1}{6} K u_{c}^{2}\left(1+\xi+\xi^{2}\right)=w,
\end{aligned}
$$

so that by identifying the other two models to Tvergaard's, gives for their material constants

$$
\begin{aligned}
& \text { Allix-Ladevèze } \quad u_{c}=\frac{27}{32} \frac{\delta}{1-\xi^{3}} \quad K u_{c}=\frac{16}{27}(1-\xi) E, \\
& \text { Cangémi et al. } \quad w=\frac{1}{12} E \delta \quad c=\frac{64}{243} E / \delta .
\end{aligned}
$$

In the applications in Figs. 26 and 27, we have taken the following values: $E=3000 \mathrm{MPa}, \delta=0.2 \mu \mathrm{m}$; $\xi=0, u_{c}=0.169 \mu \mathrm{m}, K u_{c}=1778 \mathrm{MPa} ; w=50 \mathrm{~J} / \mathrm{m}^{2}, c=3950 \mathrm{~N} / \mathrm{m}^{3}$.

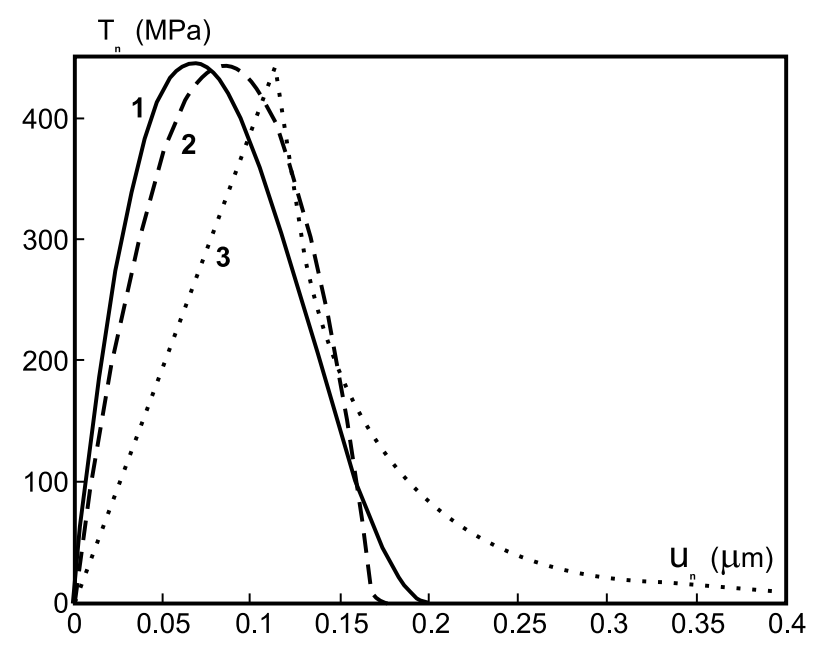

Fig. 26. Adjusted responses of the three rate independent versions: (-): Tvergaard model, (- - -): Allix-Ladevèze model, ( .): Cangémi et al. model. 


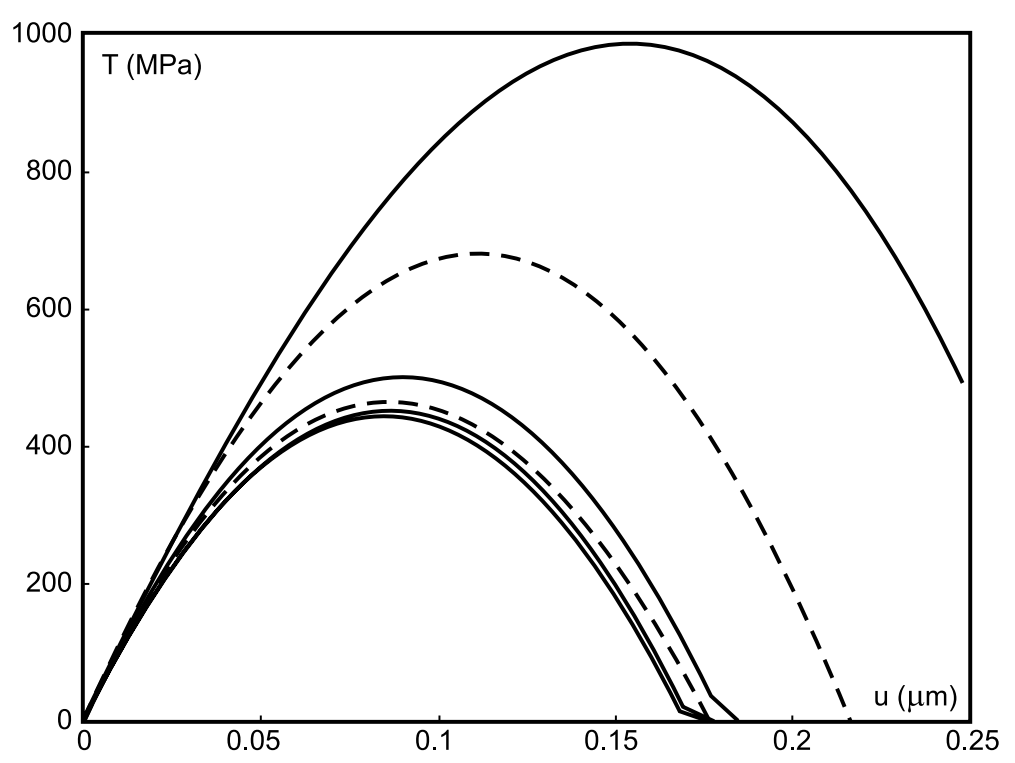

Fig. 27. The responses of the Allix-Ladevèze model for various rates of controlled displacement: $(-): \dot{u}=10^{-3}, 0.1,1,10 \mu \mathrm{m} / \mathrm{s}$ and $(\cdots): \dot{u}=0.4,4 \mu \mathrm{m} / \mathrm{s}$.

\section{C.2. Rate dependent case}

We consider the tension-displacement responses under controlled displacement at constant rate: $u=\dot{u}$. In the two models given in Appendix B, the solution cannot be extracted in closed form. Figs. 27 and 28 show responses obtained numerically, with a simple step by step implicit scheme. The model parameters

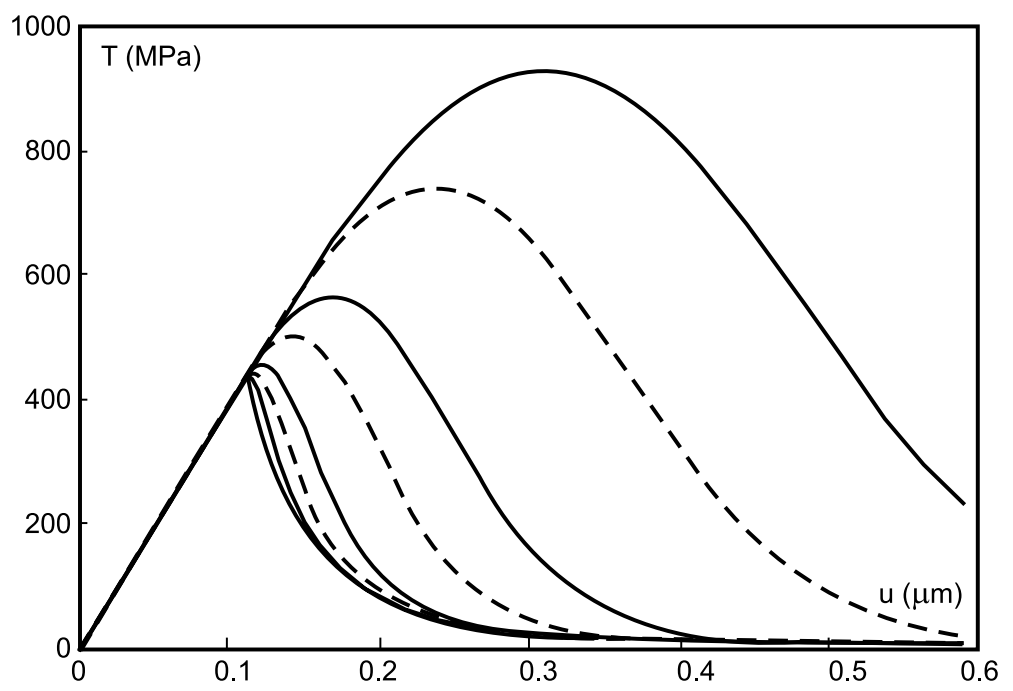

Fig. 28. The responses of the model by Cangémi et al. for various rates of controlled displacement: $(-): \dot{u}=10^{-4}, 0.01,0.1,1,10 \mu \mathrm{m} / \mathrm{s}$ and $(\cdots): \dot{u}=0.04,0.4,4 \mu \mathrm{m} / \mathrm{s}$. 
have been indicated above for their rate independent part. In addition, we selected $a=1, \eta=10^{-4} \mathrm{~s}^{-1}$ and $b=10 \mathrm{MPa} \mathrm{ms}$. Above the inviscid solution, we observe a high rate dependency in both models, both for the maximum tension and for the displacement at complete separation. Fig. 8 in Section 4 , plots the maximum traction as a function of the applied displacement rate.

Nevertheless, some asymptotic expressions may appear. In the Allix-Ladevèze model, if we assume the very high-rate situation, we saturate $\dot{d}$ to its limit value $\eta / a$, so that we can replace $d$ in Eq. (C.3) by $d=\eta u / a \dot{u}$. The condition $\mathrm{d} T / \mathrm{d} u=0$ (with a constant $\dot{u}$ ) leads to the approximate expression for the maximum tension.

$$
T_{\max } \approx \frac{1}{4} K \frac{a}{\eta} \dot{u}
$$

The corresponding line is plotted in Fig. 8. For the Cangémi model, in the high rate domain, we can neglect the threshold $w$. The damage rate equation (B.8) then expresses

$$
\dot{d}=\frac{c}{b}(1-d) u^{2}
$$

and can be integrated analytically for a constant rate $\dot{u}$ :

$$
d=1-\exp \left(-\frac{c}{3 b} \frac{u^{3}}{\dot{u}}\right)
$$

Reported in the tension-displacement relation (B.6a), we find

$$
T=c(1-d)^{2} u=c u \exp \left(-\frac{2 c}{3 b} \frac{u^{3}}{\dot{u}}\right) .
$$

We can determine the position and the value for the maximum tension by solving $\mathrm{d} T / \mathrm{d} u=0$. We find

$$
T_{\max }=c\left(\frac{b \dot{u}}{2 c e}\right)^{1 / 3}
$$

showing a $1 / 3$ power dependency (instead of the one for the Allix-Ladevèze delay model). Fig. 8 indicates the corresponding line and compares it with the numerical solution.

A third model has been studied with a viscous regularization. It corresponds to a coupling between elastic behavior and a creep equation that is most often used for metals. The equations were recalled in Section 4.

$$
T=E(1-d) \frac{u}{\delta}, \quad \dot{d}=\frac{1}{\tau_{0}}\left(\frac{T}{A}\right)^{r}(1-d)^{-s} .
$$

Eq. (C.15b) can be integrated analytically for a given displacement $u=\dot{u}$ (constant rate). We find

$$
(1-d)^{s-r+1}=1-\left(\frac{u}{u_{r}}\right)^{r+1}, \quad \frac{u_{r}}{\delta}=\left[\frac{r+1}{s-r+1}\left(\frac{A}{E}\right)^{r} \frac{\tau_{0} \dot{u}}{\delta}\right]^{1 /(r+1)},
$$

so that the traction expresses

$$
T=E\left[1-\left(\frac{u}{u_{r}}\right)^{r+1}\right]^{1 /(s-r+1)} \frac{u}{\delta}
$$

The solution of $\mathrm{d} T / \mathrm{d} u=0$ is 


$$
\frac{u}{u_{r}}=\left(\frac{s-r+1}{s+2}\right)^{1 /(r+1)}
$$

and after replacing in Eq. (C.17), we obtain the maximum traction as a function of the displacement rate

$$
T_{\max }=E\left(\frac{r+1}{s+2}\right)^{s+2 /(r+1)(s-r+1)}\left(\frac{A}{E}\right)^{r /(r+1)}\left(\frac{\tau_{0} \dot{u}}{\delta}\right)^{1 /(r+1)} .
$$

In the application by Chaboche et al. (1997a), we have set $s=r=2$, so that Eq. (C.19) reduces to

$$
T_{\max }=E\left(\frac{9}{16} \frac{A}{E}\right)^{2 / 3}\left(\frac{\tau_{0} \dot{u}}{\delta}\right)^{1 / 3} .
$$

The corresponding line is shown in Fig. 8 with $E=3000 \mathrm{MPa}, A=130 \mathrm{MPa}, \delta=0,2 \mu \mathrm{m}, \tau_{0}=10^{6} \mathrm{~s}$. Let us note the same rate dependency, in the high rate regime, as the one for the model by Cangémi et al. (1995).

\section{References}

Allix, O., 1987. Délaminage: approche par la mécanique de l'endommagement. In: Fouet, Ladevèze, Ohayon (Eds.), Calcul des Structures et Intelligence Artificielle, vol. 1, Pluralis, pp. 39-53.

Allix, O., Ladevèze, P., 1989. Damage analysis for laminate delamination. Proc. Fifth Int. Symp. on Num. Meth. in Engng. Springer, Berlin, pp. 347-354.

Allix, O., Ladevèze, P., 1992. Interlaminar interface modelling for the prediction of laminate delamination. J. Compos. Struct. 22, 235242.

Allix, O., Ladevèze, P., 1996. Damage mechanics of interfacial media: basic aspects, identification and application to delamination. In: Voyiadjis, G.Z., Allen, D.H. (Eds.), Damage and Interfacial Debonding in Composites. Elsevier, Amsterdam, pp. 167-188.

Baaser, H., Gross, D., 1998. Damage and strain localisation during crack propagation in thin-walled shells. In: Bertram, A., Forest, S., Sidoroff, F., (Eds.), EMMC2, Mechanics of Materials with Intrinsic Length Scale, Publi. Univ. Magdeburg, Germany.

Bazant, Z.P., Pijaudier-Cabot, G., 1988. Non local damage, continuum model and localisation instability. J. Appl. Mech., ASME 55, 287-294.

Belytschko, T., Lasry, D., 1988. Localisation limiters and numerical strategies for strain-softening materials. In: Mazars, Bazant, Z.P. (Eds.), Cracking and Damage Journal, Elsevier, Amsterdam, pp. 349-362.

Benallal, A., 1991. Quelques remarques sur le rôle du couplage thermomécanique dans les phénomènes de localisation. C.R. Acad. Sci. $312(2), 117-122$.

Bigoni, D., Hueckel, 1991. Uniqueness and localization: associative and nonassociative elastoplasticity. Int. J. Solids Struct. 28 (2), 197-213.

de Borst, R., 1991. Simulation of strain localization: a reappraisal of the Cosserat continuum. Engng. Comput. 8, 317-332.

Budiansky, B., Evans, A.G., Hutchinson, J.W., 1986. Matrix fracture in fiber reinforced ceramics. J. Mech. Phys. Solids 34, $167-189$.

Burlet, H., Cailletaud, G., 1986. Numerical techniques for cyclic plasticity at variable temperature. Engng. Comput. 3, 143-153.

Cangémi, L., Cocu, M., Raous, M., 1995. Adhesion and friction model for the fiber/matrix interface of a composite. Proc. of ESDA'96 - ASME Mini Symposium Contact Mechanics and Tribology, Montpellier, p. 164.

Cangémi, L., 1997. Frottement et adhérence: modèle, traitement numérique et application à l'interface fibre/matrice. Thèse de Doctorat, Université Aix-Marseille II.

Chaboche, J.L., Girard, R., Schaff, A., 1997a. Numerical analysis of composite systems by using interphase/interface models. Comput. Mech. 20, 3-11.

Chaboche, J.L., Girard, R., Levasseur, P., 1997b. On the interface debonding models. Int. J. Damage Mech. 6, $220-257$.

Delaplace, A., Pijaudier-Cabot, G., Roux, S., 1998. Damage cascade in an interface. In: Bertram, A., Forest, S., Sidoroff, F. (Eds.), EMMC2, Mechanics of materials with intrinsic length scale. Publ. Univ. Magdeburg, Germany.

Duvaut, G., Lions, J.L., 1972. Les inéquations en Mécanique et en Physique. Dunod, Paris.

Evans, A.G., Zok, F.W., 1994. Review: the physics and mechanics of fibre reinforced brittle matrix composites. J. Mater. Sci. 29, 38573896.

Feyel, F., 1998. Application du calcul parallèle aux modèles à grand nombre de variables internes. Thèse de Doctorat, ENSMP. 
Frémond, M., 1985. Contact unilatéral avec adhérence. In: Piero, D., Maceri (Eds.), Unilateral Problems in Structural Analysis, Springer, Berlin.

Frémond, M., 1987. Adhérence des solides. J. de Mécanique Théorique et Appliquée 6 (3), 383-407.

Guichet, B., 1998. Identification de la loi de comportement interfaciale d'un composite SiC/Ti. Thèse de Doctorat, Ecole Centrale de Lyon.

Hutchinson, J.W., Jensen, H.M., 1990. Models of fiber debonding and pullout in brittle composites with friction. Mech. Mater. 9, 139163.

Ladevèze, P., 1992. Towards a fracture theory. In: Owen, D.R.J., Onate, E., Hinton, E. (Eds.), Proc. Third Int. Conf. on Computational Plasticity, Pineridge Press, Cambridge, UK, pp. 1369-1400.

Ladevèze, P., 1995. A damage computational approach for composites: basics aspects and micromechanical relations. Comput. Mech. $17,142-150$.

Lemaitre, J., 1992, A course on Damage Mechanics. Springer, Berlin.

Leroy, F.H., Auvray, M.H., Lesne, P.M., 1995. Residual thermal stresses in the pull-out specimen: A finite element calculation. J. Mater. Sci. 30, 4651-4658.

Lissenden, C.J., Herakovich, C.T., 1995. Numerical modelling of damage development and viscoplasticity in metal matrix composites. Comp. Meth. Appl. Mech. Eng. 126, 289.

Lissenden, C.J., 1996. An approximate representation of fibre-matrix debonding in non-periodic metal matrix composites. In: Voyiadjis, G.Z, Allen, D.H. (Eds.), Damage and Interface Debonding in Composites. Elsevier, Amsterdam, pp. 189.

Lo, D.C., Allen, D.H., 1994. Modelling of delamination damage evolution in laminated composites subjected to low velocity impact. Int. J. Damage Mech. 3, 378-407.

Mc Cartney, L.N., 1989. New theoretical model of stress transfer between fibre and matrix in a uniaxially fibre-reinforced Composites. Proc. R. Soc. Lond. A 425, 215-244.

Marshall, D.B., Oliver, W.C., 1987. Measurement of interfacial mechanical properties in fiber-reinforced ceramic composites. J. Am. Ceram. Soc. 78 (8), 542-548.

Michel, J.C., Suquet, P., Thébaud, F., 1994. Une modélisation du rôle des interfaces dans le comportement des composites à matrice métallique. Revue Européenne des Eléments Finis 3 (4), 573-595.

Monerie, Y., Raous, M., Leroy, F.H., Sudre, O., Feyel, F., Chaboche, J.L., 1998. Comparaison de lois d'interface fibre/matrice sur la base d'un modèle simplifié d'expérience de micro-indentation, JNC 11, Bordeaux.

Murakami, H., Kendall, D.M., Valanis, K.C., 1993. A non-local elastic damage theory: mesh-insensitivity under strain softening. Comput. Struct. 48 (3), 415-422.

Nairn, J.A., 1992. A variational mechanics analysis of the stresses around breaks in embedded fibers. Mech. Mater. 13, 131-154.

Needleman, A., 1987. A continuum model for void nucleation by inclusion debonding. J. Appl. Mech. 54, 525-531.

Ousset, Y., Roudolff, F., 1997. Numerical analysis of delamination in multilayered composite plates. IUTAM Symp., Innovative Computational Methods for Fracture and Damage, vol. 20, Dublin, pp. 122-126.

Point, N., Sacco, E., 1996. A delamination model for laminated composites. Int. J. Solids Struct. 33 (4), $483-509$.

Saanouni, K., Chaboche, J.L., Lesne, P.M., 1989. On the creep crack-growth prediction by a non local damage formulation. Eur. J. Mech. 8 (6), 437-459.

Suquet, P., 1987. Discontinuities and plasticity. In: Moreau, J.J., Panagiotopoulos, P.D. (Eds.), Nonsmooth Mechanics and Applications. Springer, Berlin, pp. 280-340.

Tvergaard, V., 1990a. Effect of fibre debonding in a whisker-reinforced metal. Mater. Sci. Engng. A 125, $203-213$.

Tvergaard, V., 1990b. Micromechanical modelling of fibre debonding in a metal reinforced by short fibres. In: Dvorak, G.J. (Ed.), Proc. of the IUTAM Symp. on Inelastic Deformation of Composite Materials, Springer, Berlin, pp. 99-111. 